\title{
From Patients to Platelets and Back Again: Pharmacological Approaches to Glycoprotein VI, a Thrilling Antithrombotic Target with Minor Bleeding Risks
}

\author{
Augusto Martins Lima ${ }^{1, *}$ \\ Nikolaos Stergiopulos ${ }^{1}$ \\ ${ }^{1}$ Institute of Bioengineering, École Polytechnique Fédérale de \\ Lausanne, Lausanne, Switzerland \\ 2 Institute of Physiological Chemistry and Pathobiochemistry, \\ University of Münster, Münster, Germany
}

Ana C. Martins Cavaco ${ }^{2, *}$ Rodrigo A. Fraga-Silva ${ }^{1}$ Johannes A. Eble ${ }^{2}$

\begin{abstract}
Address for correspondence Augusto Martins Lima, PhD, Institute of Bioengineering, École Polytechnique Fédérale de Lausanne, Lausanne, Switzerland

(e-mail: augusto.martinslima@epfl.ch; augustomlima@gmail.com).
\end{abstract}

\begin{abstract}
Keywords

- GPVI

- antiplatelet therapy

- drug discovery

- protein-protein interaction

Despite significant advances in the treatment of thrombogenic diseases, antiplatelet therapies are still associated with a high bleeding risk. Consequently, potential benefits of preventing thromboembolic events by pharmacological agents need to be balanced with the potential harm of inducing hemorrhage. Glycoprotein VI (GPVI) is a platelet-specific receptor, which plays a crucial role in thrombus formation. GPVI deficiency has been identified in patients who suffer from significant reduction of collagen-induced thrombus formation, with a slight tendency for mild bleeding. However, an isolated GPVI deficiency can reduce thrombus formation while not resulting in severe bleeding. Together, these observations strongly suggest that physiological hemostasis does not require GPVI, but pharmacological GPVI modulation may provide novel "bleeding-free" antithrombotic therapies. In this review, we discuss recent findings regarding the biological role of GPVI in platelet-related disorders and highlight the efforts to develop potential therapeutic strategies based on its structure, signaling pathways, and biological effects.
\end{abstract}

\section{Introduction}

Despite significant advances in the treatment of cardiovascular diseases, in 2016, over 15 millions of deaths were caused by ischemic heart disease and stroke, induced by artery thrombosis. ${ }^{1}$ Platelets, anucleate blood cells derived from megakaryocytes, play a major role in the development of cardiovascular thrombosis. As shown in - Fig. 1, following endothelial damage caused by the growth of an atherosclerotic plaque and under high shear stress conditions, platelets will adhere to the subendothelial matrix, activate, and release prothrombotic mediators, recruiting additional platelets from the bloodstream to form an aggregate. If not treated, vascular occlusion

* These authors contributed equally to this work.

may occur. ${ }^{2}$ Platelet-mediated thrombosis is not restricted to events where extracellular matrix (ECM) is exposed to the blood vessel lumen, mainly of the artery. For instance, when vessel damage reaches the adventitial layer, tissue factors expressed in smooth muscle and adventitial cells can initiate thrombin production, thereby activating platelets. Another mechanism of platelet-mediated thrombosis, independent of ECM exposure, is through endothelial cell activation, which releases ultralarge von Willebrand factor (VWF) multimers that induce platelet activation via the glycoprotein (GP) Ib receptor complex. In each of these mechanisms, plateletmediated thrombosis can result in uncontrolled clot formation and subsequently arterial or venous thrombosis. ${ }^{4}$ Antiplatelet therapy has been an effective and efficient treatment that has

(c) 2019 Georg Thieme Verlag KG Stuttgart · New York
DOI https://doi.org/ 10.1055/s-0039-1695770. ISSN 0340-6245.
March 29, 2019

accepted after revision

July 9, 2019 


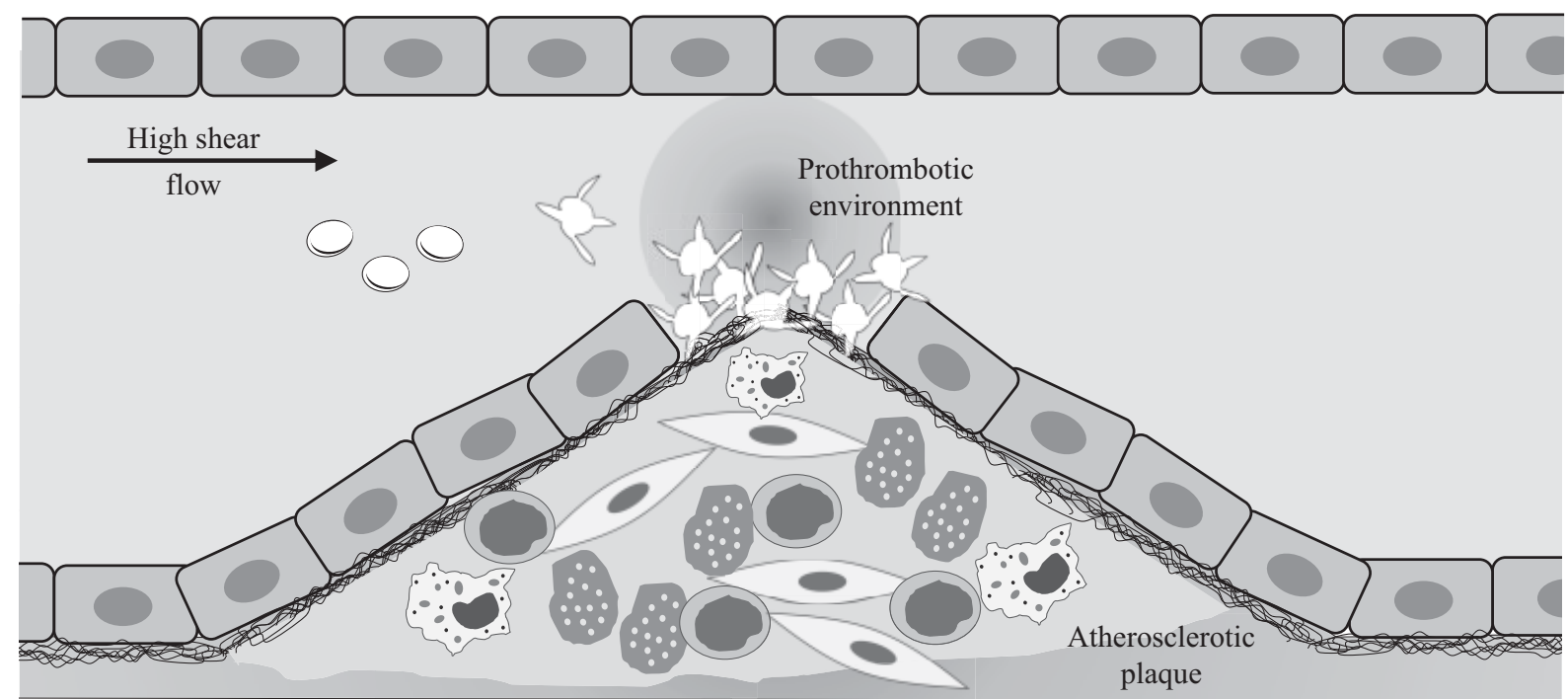

Legend
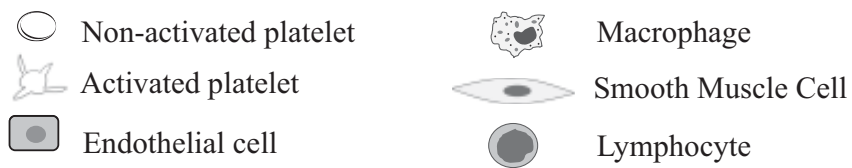

Foam Cell

Endothelial cell

Lymphocyte

Extracellular Matrix

Fig. 1 Platelet activation on atherosclerosis formation. Once an atherosclerotic plaque is established, the platelet activation process is inherently self-perpetuating. Briefly, following the damage to endothelial cells due to plaque growth, platelets will be exposed to the extracellular matrix (ECM) under a high shear stress flow condition. After adhering to ECM, platelets will become activated releasing prothrombotic factors that will recruit platelets from the bloodstream, forming an aggregate. Platelet aggregation at the injury site is crucial for thrombus formation and, if not controlled with antiplatelet drugs, may progress to vascular occlusion. ${ }^{2}$ During atherosclerotic plaque formation, other key events take place, such as rolling and adhesion of monocytes to the endothelium, supported by platelets, then transform into macrophages, and subsequently in foam cells, which produce proinflammatory cytokines. The inflammatory process accelerates as T-cells, B-cells, and dendritic cells infiltrate the plaque. ${ }^{3}$

led to reduced mortality and morbidity by targeting key pathways of platelet activation. ${ }^{5,6}$ Current antiplatelet therapy includes antagonists for adenosine diphosphate (ADP) receptors (clopidogrel, ticlopidine, prasugrel), integrin $\alpha$ IIb $\beta 3$ inhibitors (abciximab, eptifibatide, tirofiban), phosphodiesterase inhibition (dipyridamole and cilostazol), antagonists for the thrombin receptor protease-activated receptor 1 (vorapaxar), and inhibition of thromboxane $A_{2}\left(T_{X} A_{2}\right)$ synthesis (aspirin). However, such drugs present several limitations, including weak inhibition of platelet function, only a modest effect in primary prevention, and, most importantly, increase in bleeding events. ${ }^{2,5,7}$

Hemorrhage is a major concern in all currently available antithrombotic treatments. Due to the weak inhibitory effect of particular drugs on platelets, such as the one of aspirin, a common medical management strategy is to combine different pharmacological therapies. In a meta-analysis of 18 randomized trials comprising 129,314 patients, it was reported that dual antiplatelet therapy presents a $50 \%$ increase in risk of bleeding compared with the single agent therapy. ${ }^{8}$ In addition, the risk of hemorrhage can be more than threefold higher when triple therapy with warfarin is used to prevent and treat venous and arterial thrombosis, leading to an increase in the risk of fatal bleeding. ${ }^{9}$ Furthermore, the risk of bleeding due to antiplatelet therapy increases with age. Recently, Li et al demonstrated, in a large population study, that the longterm risks and severity of bleeding in patients receiving aspirin increases dramatically with age (older than 75 years) and with substantial risk of fatal bleeding. ${ }^{10}$ For these reasons, the potential benefit of preventing thromboembolic disease needs to be balanced with the potential harm of inducing hemorrhage. Therefore, antiplatelet therapy can be improved by developing new drugs capable of treating thrombotic events without causing bleeding as an adverse effect.

\section{The Search for Inhibitors of GPVI, a Potential Target for "Bleeding-Free" Antithrombotic Therapy}

Several studies have recently reported GPVI as a potential target for antithrombotic therapy, which would not affect the physiological homeostasis. ${ }^{11,12}$ Remarkably, reduction of thrombus formation by collagen ex vivo was observed in a patient with GPVI deficiency. ${ }^{13}$ Additionally, the GPVI-receptor pathway is relevant in human cardiovascular disease, as the expression level and activity of GPVI correlates with the development of transient ischemic attack, ${ }^{14}$ stroke, ${ }^{14}$ acute coronary syndrome, ${ }^{15}$ and myocardial infarct. ${ }^{16}$ These data are in accordance with observations that GPVI inhibition leads to reduction of thrombus formation. ${ }^{17-20}$

Furthermore, GPVI deficiency was identified in patients who have, at the most, a slight tendency for mild bleeding, ${ }^{1321-32}$ suggesting that isolated GPVI deficiency does not result in severe bleeding. ${ }^{11}$ Similarly to humans, 
GPVI-deficient mice demonstrated no impact on bleeding time, but collagen-induced platelet activation and thrombus formation is dramatically reduced. ${ }^{17,33}$ These observations strongly suggest that physiological hemostasis does not require GPVI. This supports the concept that pharmacological modulation of GPVI can open novel strategies for "bleeding-free" antithrombotic therapies. Finally, GPVI not only mediates platelet activation at the site of vascular injury where collagen is exposed, but also is implicated in the pathogenesis of other disorders, such as rheumatoid arthritis $^{34}$ and tumor metastasis. ${ }^{35}$ These results suggest that pharmacological targeting of GPVI may lead to beneficial effects in a wide range of platelet-related diseases.

In summary, GPVI is a potential target for antithrombotic therapy because:

1. GPVI is only expressed in platelets and megakaryocyte (platelet precursor cells), ${ }^{36}$ potentially leading to high cell specificity and reduced side effects.

2. GPVI inhibition decreases collagen-induced platelet function with minimal impact on bleeding.

3. The beneficial effect of GPVI inhibition on cardiovascular diseases is well-documented (for more information see review Andrews et al). ${ }^{12}$

\section{Role of GPVI on Platelet Function and Hemostasis}

Several studies have reported the effect of GPVI deficiency on human platelet function and their main findings are summarized in the - Table 1. In 1987, the first case of human GPVI deficiency was reported, indicating the important role of GPVI on platelet function. ${ }^{21}$ The subsequent studies using the patient's blood were crucial to expand the knowledge about GPVI by demonstrating: (1) the involvement of spleen tyrosine kinase (Syk) in GPVI intracellular signaling ${ }^{37,38}(2)$ the validation of collagen-related peptide (CRP) as a GPVIspecific agonist ${ }^{39}$ and (3) the crucial role of GPVI on platelet degranulation induced by collagen. ${ }^{39}$ Next, additional studies, using GPVI-deficient human platelets, have demonstrated not only the importance of GPVI as a collagen receptor, but also that its absence leads, in the most severe cases, to mild bleeding (average bleeding time measured by the Duke's method - GPVI-deficient patients: 9 minutes vs. normal subjects: 3-5 minutes). ${ }^{21,24,26,27,31}$ Importantly, several reports have shown thrombus formation is dramatically reduced when human platelets lack GPVI, indicating the GPVI receptor as a potential target for inhibition of thrombus formation, with a minimum impact on patients' bleeding time. ${ }^{13,22,25,28}$ Here, it is important to highlight that the beneficial effect of preventing bleeding by GPVI inhibition might be either underestimated by the presence of other platelet-related disorders, such as thrombocytopenic purpura, systemic lupus erythematosus, and gray platelets syndrome, which affects the count and the structure of platelets and contributes to a longer bleeding time or overestimated due to the fact that FCRyIIA, a major FcR expressed only in higher primates, is most likely, activated in patients with GPVI deficiency induced by shedding.
It is well documented that FcyRIIa interacts with several platelet receptors and it has been reported that the crosslinking of GPVI with FcrRIla seems to be important for platelet activation (for more information see review by Qiao et $\mathrm{al}^{43}$ ). In addition, the activation of FcrRIIa induces metalloproteinases-mediated ectodomain shedding of $\mathrm{GPVI}^{30}$ Based on these findings, one can speculate that the bleeding-free outcome induced by GPVI inhibition might be due to FcrRIla activation, which could explain the pathological mechanism of patients with GPVI deficiency induced by shedding. Studies which aimed to clarify the importance of FcyRIIa on GPVI-induced platelet activation are contradictory, to date. For example, the antibody OM1, induces GPVI-dependent platelet aggregation without the requirement of FcrRIIa, whereas other GPVI antibodies, OM2 and 9012.2, activated platelets in an FcrRIIadependent manner. ${ }^{44,45}$ In addition, a recent report demonstrated that spreading and adhesion on fibrin is dramatically decreased in GPVI-deficient mouse platelets but are not altered in human platelets treated with monoclonal FcrRIIa-inhibiting antibody IV.3. ${ }^{46}$ Therefore, further studies are needed to understand whether the beneficial antibleeding effect of GPVI inhibition is independent of FcrRIIa activation. This could be assessed by using, for example, platelets from GPVI-deficient patients.

Although recent studies have speculated that fibrin(ogen) is a potential GPVI agonist, platelets from GPVI deficiency patients were not tested upon stimulation with fibrin(ogen). Apart from one study in which platelets were reported to lack $\alpha$-granules, ${ }^{29}$ platelet aggregation and degranulation induced by non-GPVI agonists and the expression of other platelet receptors are preserved in patients with GPVI deficiency. In contrast, the aggregation response induced by the GPVI agonists, collagen-I, CRP, and convulxin (CVX), is dramatically impaired. In most cases, platelet aggregation is abolished in response to CRP and CVX, and it is reduced at high concentrations of collagen-I ( $>10 \mu \mathrm{g} / \mathrm{mL})$. These findings corroborate several studies in GPVI-knockout animals where platelet aggregation was reduced with a minor effect on platelet adhesion and a reduction of thrombus formation without any increase of bleeding time. ${ }^{17,33}$ Collagen-induced platelet aggregation at high concentrations is only abolished when platelets are preincubated with $\alpha 2 \beta 1$-integrin inhibitor. $^{22}$ Collagen-I also binds to $\alpha 2 \beta 1$ integrin, and activation of this receptor triggers platelet aggregation, although it requires much longer time to reach the maximal aggregation, when compared with collagen or $\mathrm{CVX} .{ }^{47}$ In addition, human deficiency of $\alpha 2 \beta 1$ integrin on platelets results in severe bleeding, indicating a crucial role of this platelet collagen receptor for maintenance of hemostasis, and less relevance in platelet aggregation. ${ }^{48-51}$

Taken together, these results demonstrated that GPVI is a key mediator of collagen-induced platelet aggregation and, differently from $\alpha 2 \beta 1$ integrin, GPVI deficiency does not cause severe bleeding. In addition, the lack of GPVI does not interfere in platelet function (aggregation, adhesion, and degranulation) if induced by the activation of non-GPVIspecific platelet agonists. 


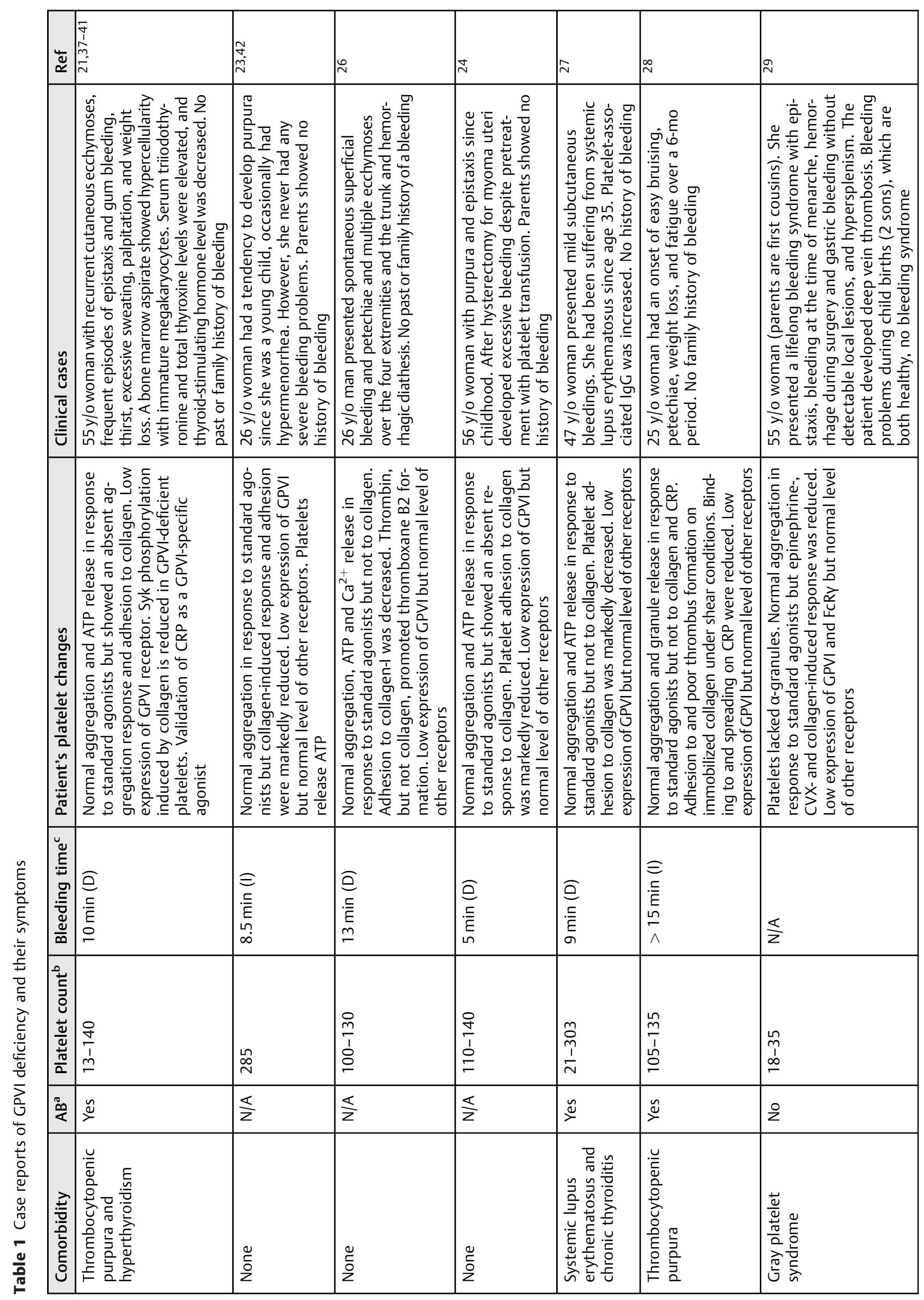




\begin{tabular}{|c|c|c|c|c|c|}
\hline$\stackrel{\bar{\Xi}}{\simeq}$ & & m & 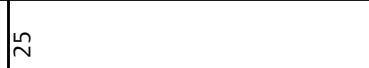 & $m$ & $\bar{m}$ \\
\hline 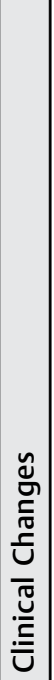 & 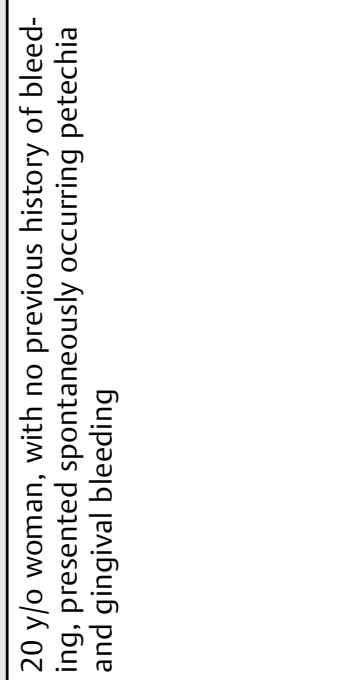 & 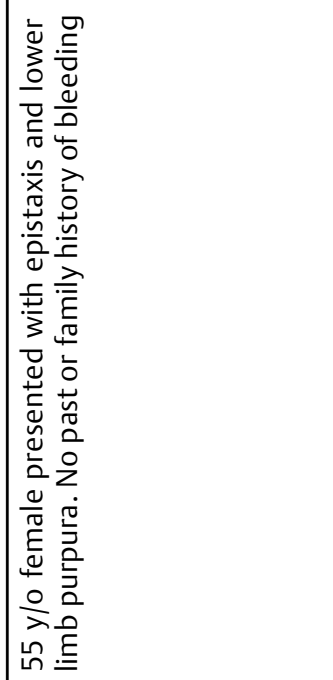 & 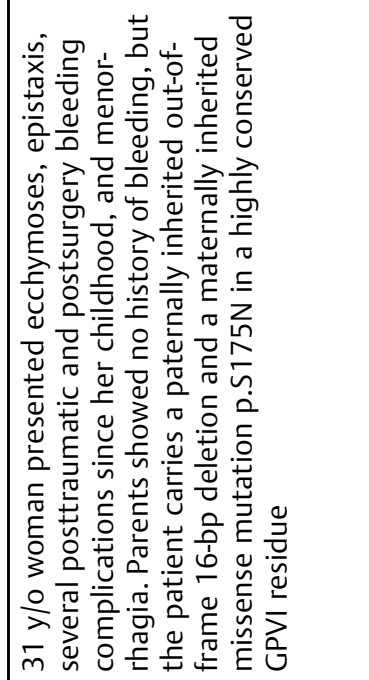 & 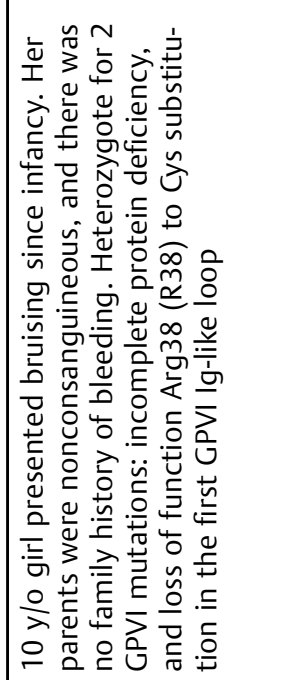 & 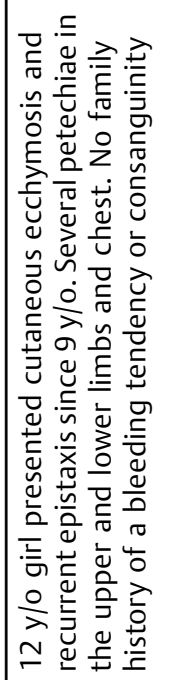 \\
\hline 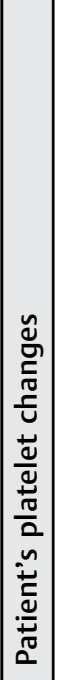 & 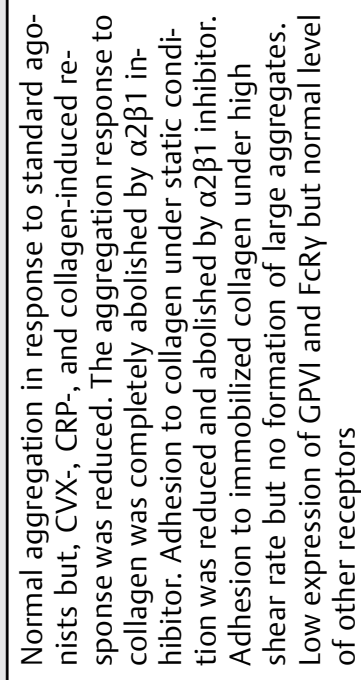 & 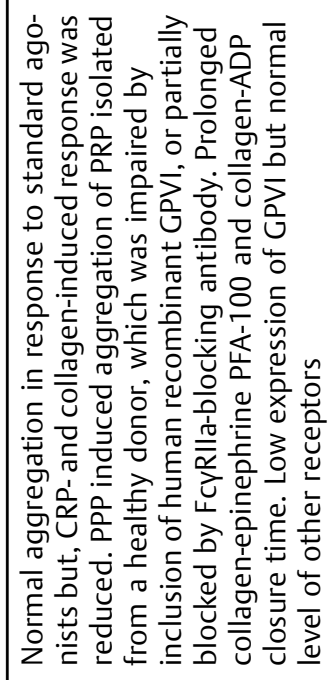 & 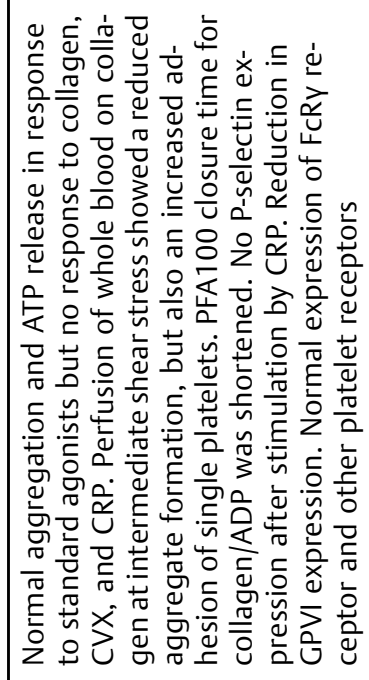 & 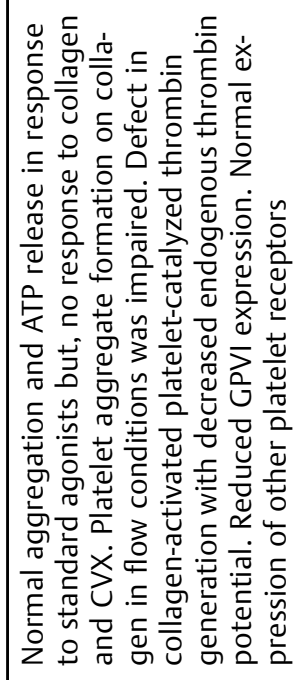 & 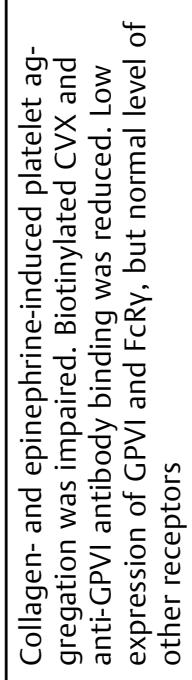 \\
\hline 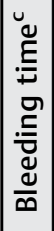 & 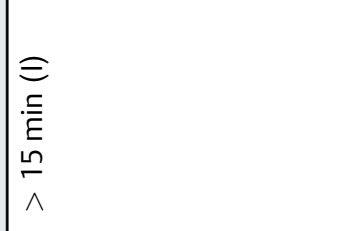 & $\frac{\ll}{z}$ & $\frac{s}{z}$ & 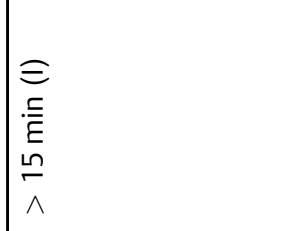 & 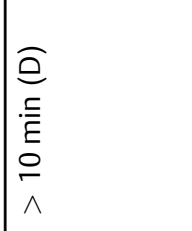 \\
\hline 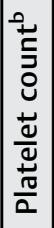 & $\stackrel{\infty}{+}$ & $\sim$ & $\stackrel{\infty}{\sim}$ & 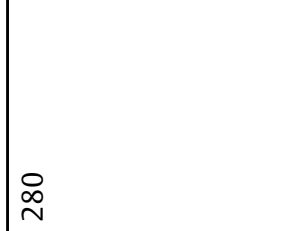 & $\infty$ \\
\hline 离 & 은 & $\stackrel{\check{\nu}}{\check{\nu}}$ & $\frac{\pi}{z}$ & $\frac{s}{z}$ & 운 \\
\hline 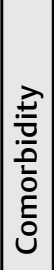 & 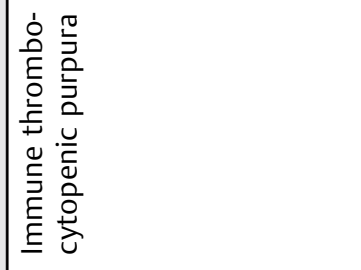 & 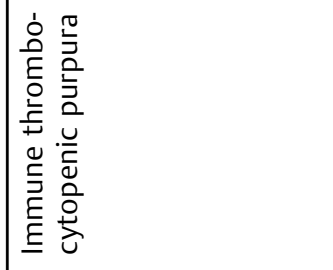 & $\begin{array}{l}\tilde{\check{L}} \\
\text { Z }\end{array}$ & 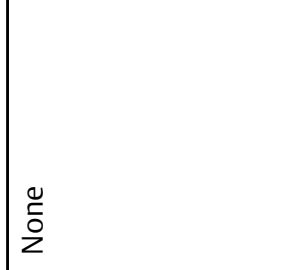 & $\mid \begin{array}{l}0 \\
\text { Oे } \\
z\end{array}$ \\
\hline
\end{tabular}




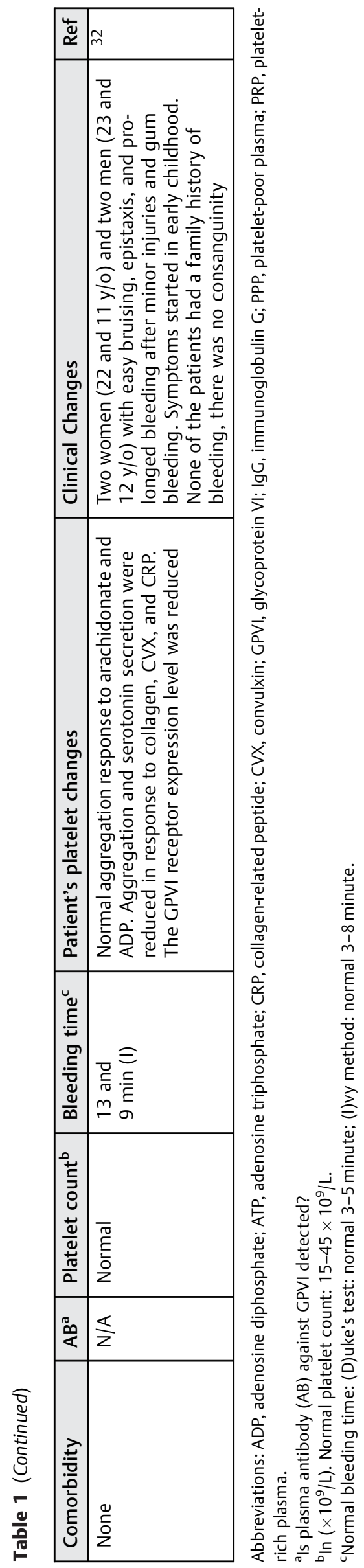

\section{Biochemical Structure of GPVI}

According to its complementary deoxyribonucleic acid cloning, in humans, GPVI consists of 319 amino acids residues, with a 20-amino-acid signal sequence. The GPVI gene presents eight exons and is located in the chromosome $19 q 13.4$ of the human genome. ${ }^{52,53}$

GPVI receptor is included in the immunoglobulin (Ig)-like receptor family, since its extracellular region possesses two Ig-like domains, designated D1 and D2, and a mucin-like Ser/ Thr-rich region. ${ }^{52,54}$ The domains D1 and D2 are linked by a single peptide strand that allows limited flexibility in the head of the receptor and a glycosylated stem connects D2 to the transmembrane domain. ${ }^{54}$ One potential glycosylation site for the $\mathrm{N}$-linked carbohydrate chain was identified at R272 and many O-linked carbohydrate chains are conjugated to the Ser/Thr-rich region. ${ }^{55}$ Therefore, it is possible that the Ig-like domains extends and tower above the glycocalyx polysaccharide layer of the platelet surface, structurally similar to GPIb/V/IX. ${ }^{52}$

The cytoplasmic domain of GPVI, consisting of 51 amino acids in humans, has two unique sequences: a basic aminoacid-rich region near the transmembrane region and a proline-rich motif in the middle of the cytoplasmic region. ${ }^{52,54}$ While the basic amino-acid-rich region binds to calmodulin, ${ }^{56}$ the proline-rich sequence selectively binds to the proto-oncogene tyrosine-protein kinase ( $\mathrm{Src}$ ) homology 3 (SH3) domain of the Src family tyrosine kinases Fyn and Lyn. ${ }^{57}$ The C-terminal region seems to be irrelevant for GPVI function, since the cytoplasmic region of mouse GPVI contains only 27 residues and lacks the additional 24 residues which make up the C-terminal sequence of human GPVI. $^{52}$

As summarized in - Table 2, several site-directed mutagenesis studies were performed, revealing crucial amino acids residues for GPVI-agonist binding. Mutations at residues V34, K59, R60, and R166 markedly decreased the binding of recombinant GPVI to collagen or CRP, whereas N92, S94, and R272 decreased the cell response to CVX and CRP. In contrast, mutation of lysine 41 to alanine increases the binding of GPVI to both CRP and collagen. The importance of these amino acids residues for binding to collagen needs to be confirmed by crystallography. In 2006, Horii et al revealed its crystal structure and thus advanced our understanding of the GPVI receptor and its putative molecular mechanism of collagen binding. Moreover, the molecular structure of the receptor confirmed previously published data and, in addition, disclosed that two GPVI molecules associate into a backto-back dimer. The drawback of this study is the fact that the GPVI-ligand complex was predicted by computational docking, only the noncrystal structure of the complex was presented. ${ }^{63}$ Recently, an additional three crystal structures were added in the Protein Data Bank database (ID: 5OU7, 5OU8, and 5OU9) demonstrating GPVI-collagen-peptide complexes, which provided essential information to identify the collagen binding site within the GPVI receptor. ${ }^{64}$ Unfortunately, all data and methodology related with these new complexes have yet to be published. 
Table 2 Site-directed mutagenesis studies using GPVI receptor

\begin{tabular}{|l|l|l|}
\hline Point of mutations & Effect & Ref \\
\hline $\begin{array}{l}\text { K59, R60, F91, R117, } \\
\text { S118, F120, R139, }\end{array}$ & $\begin{array}{l}\text { Mutation of K59 markedly decreases the binding of GPVI to CRP. Triple } \\
\text { mutation (K59E, R117P, R166S) induced the most profound reduction of } \\
\text { binding to CRP }\end{array}$ & 58 \\
\hline $\begin{array}{l}\text { K41, R60, R166, K59, } \\
\text { F120, S164 }\end{array}$ & $\begin{array}{l}\text { Mutations of R60 and R166, as well as K59, reduce binding of GPVI to } \\
\text { collagen, whereas K41 increases the binding of GPVI to both CRP and } \\
\text { collagen }\end{array}$ & 59 \\
\hline G30, V34, L36 & $\begin{array}{l}\text { V34 strongly impairs GPVI interaction with collagen and CRP, whereas } \\
\text { L36A mutation slightly reduces the binding capacity. Triple mutation } \\
\text { (G30, V34 and L36) fully inhibits the binding to collagen and CRP (but not } \\
\text { to CVX). None of the recombinant GPVI mutations affects the maximal } \\
\text { binding to the GPVI antibody 9012 }\end{array}$ & 60 \\
\hline N92, S94, L95 & $\begin{array}{l}\text { Mutation of N92 and S94 dramatically reduces the adhesion of trans- } \\
\text { fected-cells to CVX and CRP. Whereas mutation of L95 does not change } \\
\text { the adhesion to GPVI-specific agonists. N-linked glycosylation at the } \\
\text { consensus site Asparagine92-Glycine-Serine94 of human GPVI and the } \\
\text { presence of complex oligosaccharides at this position is important for } \\
\text { maximum binding of GPVI to both CVX and CRP }\end{array}$ & 61 \\
\hline R272, W291, H292, & $\begin{array}{l}\text { Mutation of R272 decreases the Ca2+ release in response to CVX in } \\
\text { transfected cells. In addition, cells expressing the R272-mutated GPVI are } \\
\text { unable to co-precipitate with FCR y-chain }\end{array}$ & \\
\hline C274, C338 & C338 is important for GPVI dimer formation in CVX-stimulated cells & 62 \\
\hline
\end{tabular}

Abbreviations: CRP, collagen-related peptide; CVX, convulxin; GPVI, glycoprotein VI.

\section{GPVI Dimerization and Binding Specificity}

Initially, GPVI was described to form a complex with the Fc receptor $(\mathrm{FcR}) \mathrm{r}$-chain, which is expressed on other hematopoietic cells. ${ }^{65}$ Complex formation with the FcR $\gamma$-chain is essential for GPVI signaling. ${ }^{66}$ However, whether GPVI is exposed on the cell surface independently of complex formation is not clear. Some studies, where GPVI and FcR $\gamma$-chain were transfected into human cells, showed that GPVI is expressed on the cell surface, independently of complex formation, but is unable to signal on its own. ${ }^{66}$ The interaction between GPVI and the FcRr-chain depends on a salt bridge between the charged amino acid, R272, located in the transmembrane domain and a negatively charged aspartic acid residue, residing in the transmembrane domain of the FcR $\gamma$-chain. ${ }^{55}$ The GPVI receptor appears on the platelet surface, also in its oligomeric form, then each GPVI molecule is in complex with the FcR $\gamma$-chain. ${ }^{67}$ In addition, a monomeric GPVI exhibits low affinity for collagen when compared with a dimeric GPVI, ${ }^{68}$ which may explain why the oligomeric form of this complex occurs on the surface of platelets.

Collagen is an important GPVI ligand. In the vasculature, different collagens have been detected, including: collagen types I, III-VIII, XIII-XVI, XVIII, and XIX. ${ }^{69}$ However, only some collagen types bind to GPVI, such as the large fibril-forming collagen I, II, and III. To evaluate collagen IV as a ligand may prove to be difficult as it is a component of the complex basement membrane (BM), which is composed of a variety of proteins, and it is present as a thin sheet-like structure. ${ }^{70}$ The reduced affinity of collagen $V$ to GPVI can be explained by either the small size of the collagen $V$ fibrils not covering the binding site of the GPVI dimer properly ${ }^{71}$ or due to its high content of glycosylated hydroxylysine residues within the triple helical molecule portion which may impair their interaction. ${ }^{72}$ The adhesion of GPVI to collagens IV and V has so far provided confounding results. Some authors have described platelets from patients lacking the GPVI not adhering to bovine collagens IV and V. ${ }^{23,73}$ Others have observed adhesion to these collagens, but they attributed it to possible contamination of the preparation with other collagens, such as collagen-I. ${ }^{71}$ Yet, another study demonstrated that type IV and type $V$ collagens did not have any affinity for GPVI. ${ }^{52}$ Nonetheless, a role in GPVI-dependent platelet activation, at superficial vascular injury sites, has been attributed to collagen IV. ${ }^{74}$

GPVI-collagen interaction and platelet activation occur specifically through the amino acid motif glycine-prolinehydroxyproline (GPO), which comprises approximately $10 \%$ of the triple helix in collagen molecules. ${ }^{75}$ The observation that 5 or more GPO repeats spontaneously align into triple helical structures that are similar to tissue collagen molecules prompted the development of synthetic peptides. ${ }^{76}$ Moreover, such triple-helical collagenous molecules, with three chains of repetitive GPO motifs, were laterally bundled by covalently cross-linkage of their $\mathrm{N}$ - and C-terminal cysteine or lysine residues, forming the so-called CRP, a powerful synthetic platelet agonist, specifically binding to GPVI. ${ }^{77}$

During collagen-dependent platelet activation, multiple GPVI binding sites are clustered. Furthermore, under flow conditions, platelet adhesion to collagen-I depends on interaction with other collagens, such as collagen-III, indicating that ligand interactions may support platelet receptor clustering. ${ }^{71}$ Another study has shown that not only integrin $\alpha 2 \beta 1,{ }^{47}$ but also GPVI form clusters along collagen fibers, 
with the homotrimeric collagen-III being the most effective substratum for cluster formation. ${ }^{78}$

Both fibrinogen and fibrin have recently been described as GPVI ligands. Several studies demonstrated that fibrin and fibrinogen induce platelet signaling, as well as support thrombus formation and stabilization. ${ }^{79} \mathrm{~A}$ marked increase of tyrosine phosphorylation, including in the $\mathrm{FcRr}$ chain and Syk in human platelets, increased calcium $\left(\mathrm{Ca}^{2+}\right)$ signaling, as well as attachment and spreading of platelets, have been observed in response to fibrin(ogen), supporting the hypothesis that fibrin(ogen) is a potential GPVI ligand, in addition to binding to the platelet fibrin receptor, integrin $\alpha \operatorname{Ilb} \beta 3{ }^{80,81}$

Among other endogenous ligands, the adipocyte-derived cytokine, globular adiponectin (gAd), was also proposed to bind to GPVI. This interaction leads to rapid aggregation of platelets, in a tyrosine kinase-dependent mechanism. ${ }^{82}$ Studies using FcRr null mouse platelet showed that aggregation by gAd was abolished in platelets, and the authors concluded that gAd may promote adverse platelet activation at sites of vascular injury. ${ }^{82}$ However, the functional relevance of this putative GPVI-gAd interaction has remained elusive, since circulating levels of gAd are not sufficient to mediate platelet activation. ${ }^{70}$

Laminin-411 and -511 are major components of the blood vessel BM. These laminin isoforms might be additional endogenous ligands of GPVI. Isolated laminin from human placenta, with a high content of laminin-511, supported the spreading of platelets through a GPVI-dependent mechanism. ${ }^{83}$ This interaction depends on initial integrin $\alpha 6 \beta 1$ adhesion to laminin. ${ }^{83}$ The authors suggested that GPVI interaction with laminin might contribute to platelet spreading in vivo. Inoue et al also observed that platelets adhere to laminin under shear conditions, although forming smaller aggregates than the platelets that adhered to collagen, which the authors explained by the fact that GPVI has lower affinity for laminin as compared with collagen. ${ }^{84}$ The mechanism of shear-resistant attachment of platelets to laminin depends on the interaction of VWF and GPIb-IX-V. ${ }^{84}$ Another study by Schaff et al attributed to GPVI yet another important function, not only in the adhesive process, but in the mediation of platelet activation induced by $\alpha 5$-containing laminins. ${ }^{85}$ Consequently, the study concluded that laminins contribute to thrombus formation in vivo. This latter interaction could be critical for vessel repair following minor damage, without the risk of forming occlusive thrombi. Indeed, after mild injury, where the sporadic detachment of an endothelial cell can occur, these subendothelial BM proteins are the first ECM components that platelets come into contact with. ${ }^{70}$ However, in the prevention of major bleeds occurring after severe damages reaching far beyond the vessel wall, laminins might be irrelevant for platelets' thrombotic functions, as it induced only a weak activation of GPVI, as compared with the fibrillary collagens of the interstitial stroma. ${ }^{70}$

\section{Intracellular Signaling Downstream of GPVI}

Firm platelet adhesion to collagen and fibrin via their respective integrin receptors requires prior integrin activation through "inside-out" signals, generated by GPVI signaling and reinforced by the release of ADP and $\mathrm{TXA}_{2}{ }^{76}$

Platelet signaling is initiated through the immunoreceptor tyrosine-based activation motif (ITAM) that consists of two YXXL motifs, separated by $6-12$ residues, ${ }^{86}$ in the cytosolic domain of several signaling receptors. The FcRr chain harbors such an ITAM motif. Other platelet receptors, such as CLEC-2, contain only one singular YXXL motif, termed (hem)ITAM, but complement the two hemITAM motifs, resulting in a fully functional ITAM structure, by dimerization of two molecules. The GPVI-FcRr complex is critical for the signaling activity of platelets and thrombus formation, similar to other immune and C-type lectin receptors. ${ }^{87,88}$ Moreover, FcrRIIA mediates platelet integrin $\alpha I I b \beta 3$ "outside-in" signaling, thus contributing to general thrombus stabilization. ${ }^{89}$

The cross-linking of GPVI triggers the phosphorylation of the two cytosolic motifs in the FcR $\gamma$-chain ITAM by Src family tyrosine kinases, leading to binding and activation of the two tandem Src Homology 2 (SH2) domains by Syk. Consequently, this triggers the downstream signaling cascade that culminates in the activation of phospholipase $\mathrm{C}_{2} 2$ (PLC 2 2). ${ }^{90}$ However, prior to activation of $\mathrm{PLC}_{2} 2$, various adapter and effector proteins are recruited. The ITAM phosphorylation occurs in the cholesterol-rich membrane lipid rafts, that are enriched in essential GPVI signaling molecules. ${ }^{91}$ Interestingly, studies using rat GPVI-expressing RBL$2 \mathrm{H} 3$ cells and human platelets, have shown that the GPVIFcRr complex does not constitutively associate with rafts, but upon ligand stimulation, it is recruited to lipid rafts. ${ }^{91}$ Within lipid rafts, an essential protein for the formation of the signalosome can be found, which is the linker for activation of T cells (LAT).$^{92}$ However, some studies have shown that phosphorylation of Syk, LAT, and PLC 22 does not depend on the integrity of the lipid rafts in human platelets. ${ }^{93}$ The Src kinases, Fyn and Lyn, are also present in rafts and bind to a conserved proline-rich domain in the juxtamembrane region of GPVI, resulting in the phosphorylation of the FcR $r$ immune receptor ITAM sequences, which are essential for triggering downstream signals. ${ }^{57}$ While Lyn binds preferentially to the proline-rich domain and is required for rapid cell adhesion to collagen at high shear, Fyn does not bind to this domain, as it seems to be more important to sustain than to initiate the signaling events. ${ }^{94}$ Studies by Quek et al which knocked out Lyn, Fyn, or both in mouse models further revealed that, although Fyn deficiency apparently does not affect the tyrosine phosphorylation of PLC $\gamma 2$, its activity is significantly reduced, possibly due to the fact that phosphorylation of adapter proteins, such as LAT, was diminished. This resulted in reduced recruitment of $\mathrm{PLC}_{2} 2$ to the membrane. ${ }^{95}$

The activation of tyrosine kinase Syk leads to the phosphorylation of tyrosine on the adapter protein LAT. ${ }^{70}$ After phosphorylation, LAT recruits the SH2-containing adapter proteins Grb2 and Gads, which constitutively associate with SLP-76. ${ }^{96}$ PLCY2 binds to the complex, through adapter proteins LAT and SLP-76, and generates the second messengers, $\mathrm{Ca}^{2+}$ and diacylglycerol (DAG). ${ }^{87}$ Syk also plays an essential role in activating $\mathrm{PLC} \gamma 2$, since it phosphorylates SLP-76. ${ }^{97}$ While SLP-76 is essential for tyrosine 
phosphorylation and activation of the phospholipase, the absence of LAT resulted in a limited degree of tyrosine phosphorylation activation of PLC $\gamma$, but it is unclear whether LAT is required for full platelets activation. ${ }^{98}$

Additional adapter and effector proteins were identified to assemble into the ITAM signalosome, such as the signal transducer and activator of transcription 3 (STAT3), ${ }^{99}$ the Tec family tyrosine kinases Btk ${ }^{100}$ and Tec, ${ }^{101}$ the small $G$ protein Rac1, ${ }^{102}$ the GTP exchange factors Vav1 and Vav3, ${ }^{103}$ the ubiquitinating protein, $\mathrm{c}-\mathrm{Cbl},{ }^{104}$ and the $\alpha$ and $\beta$ isoforms of phosphatidylinositol 3-kinase (PI3K). ${ }^{105}$ All these proteins are involved in the signaling process within the platelet. Upon collagen stimulation, platelets activate STAT3, which serves as a protein scaffold to facilitate the catalytic interaction between the Syk kinase and its substrate, PLCY2, which releases further secondary messengers. ${ }^{99}$ Studies using human platelets and STAT3 null mouse platelets have indicated that STAT3 facilitates the crosstalk between proinflammatory cytokines and hemostasis/thrombosis signals in platelets. ${ }^{99}$ Btk works together with Tec, since Tec appears to have a functional role in the regulation of PLC 22 in the absence of Btk. Nevertheless, these proteins are essential prerequisites for platelet activation and aggregation by GPVI, regulation of tyrosine phosphorylation of PLC $\gamma 2$, as well as increase in $\mathrm{Ca}^{2+}{ }^{101}$ Additionally, Vav1 and Vav3, guanine nucleotide exchange factors (GEFs) for the Rho/Rac family, have a role in the activation of $\mathrm{PLC} \gamma 2{ }^{103}$ This process happens by tyrosine phosphorylation upon GPVI activation and platelet deficiency in both Vav1 and Vav3 decrease aggregation and spreading, as a consequence of reduced phosphorylation of PLCY $2 .{ }^{103}$ The $\mathrm{c}-\mathrm{Cbl}$ protein has been described to be tyrosine-phosphorylated in response to stimulation of GPVI; however, the role of this protein is not clear. Some studies have described it as an adaptor protein that promotes PI3K $\beta$ activation and consequently, activation of PLC $\gamma 2$ in a knockout mouse model. ${ }^{104}$ An earlier study showed that phosphorylation of several proteins, such as Syk and PLCY2, was increased in the absence of $\mathrm{c}-\mathrm{Cbl}$ on the surface of murine platelets, leading to the conclusion that it negatively regulates platelet responses to GPVI agonists. ${ }^{106} \mathrm{PI} 3 \mathrm{~K}-\alpha$ and $-\beta$ are involved in GPVI-mediated inositol 1,4,5-triphosphate $\left(\mathrm{IP}_{3}\right)$ production and $\mathrm{Ca}^{2+}$ mobilization, via PLC 2 , and contributes to Rap1b activation. ${ }^{105}$ Activated PLCY2 hydrolyzes phosphatidylinositol 4,5-bisphosphate, generating the second messengers DAG and $\mathrm{IP}_{3}$, which in turn, mediates the release of $\mathrm{Ca}^{2+}$, whereas DAG activates protein kinase $\mathrm{C}$ (PKC). ${ }^{107}$ The GTPase Rap1 is activated downstream of PLC 2 in a $\mathrm{Ca}^{2+}$-dependent process, where $\mathrm{Ca}^{2+}$ is sensed by GEF, CalDAG-GEFI, which will then facilitate this activation. ${ }^{87}$

The GPVI signaling pathway can be inhibited by Src kinases if a relevant tyrosine residue in an inhibitory site is phosphorylated. Hence, dephosphorylation of this site is critical for activation ${ }^{70}$ and seems to depend on the membrane tyrosine phosphatase, CD148. In the absence of this phosphatase, Src family kinase activity is reduced and GPVImediated activation is impaired, resulting in defective thrombosis. ${ }^{108}$ Lyn plays an inhibitory role in mouse platelet activation through tyrosine phosphorylation of platelet en- dothelial cell adhesion molecule- 1 and subsequent binding of the $\mathrm{SH} 2$ domain-containing phosphatase. ${ }^{109}$ Additionally, this complex suppresses GPVI signaling.

\section{CLEC-2 a GPVI Homologous Receptor}

The C-type lectin receptor 2 (CLEC-2) is a recently discovered platelet receptor that shares structural and functional similarities with GPVI. ${ }^{88}$ It is a type-II membrane protein with a C-terminal extracellular domain comprising a stalk region and a carbohydrate-like recognition domain that lacks the conserved amino acids necessary for binding carbohydrates. $^{70}$ For this reason, CLEC-2 is considered a C-type lectin-like domain-containing protein. ${ }^{110}$ The cytoplasmic $\mathrm{N}$-terminal tail of 31 amino acids, contains a single and conserved YxxL sequence, an hemITAM, starting at position 7, downstream of a tri-acidic amino acid region (DEDG). ${ }^{70}$ Similarly to GPVI, CLEC-2 is highly expressed on megakaryocytes and platelets but also at low levels on primary human liver sinusoidal endothelial cells and on neutrophils, and possibly on other hematopoietic cells. ${ }^{70}$

The human CLEC-2 gene, also known as CLEC1B, is located on chromosome 12 in a cluster with six other C-type lectin receptors. An example of this is Dectin-1, which, like CLEC-2, is a member of group $\mathrm{V}$ of the type II C type lectin-like receptors. ${ }^{70}$

CLEC-2 has an exogenous ligand, rhodocytin, a snake venom component, which upon binding to CLEC-2 triggers tyrosine phosphorylation of the CLEC- 2 hemITAM sequence and, consequently, other signaling proteins. ${ }^{111}$ The first endogenous ligand for CLEC-2 was identified by the group of Yukio Ozaki, and described as a transmembrane sialoglycoprotein, podoplanin. ${ }^{112}$ Moreover, podoplanin expressed by tumor cells mediates aggregation of platelets, in a Src kinases/ PLCr2-dependent manner, similarly to rhodocytin. ${ }^{112}$ Other authors linked podoplanin from kidney HEK293T cells to platelet activation. ${ }^{113}$ Podoplanin can be found in different tissues like brain, heart, kidney, lungs, osteoblasts, and lymphoid organs, ${ }^{114}$ but not on blood cells or platelets. ${ }^{87}$ This raises a controversy on the importance of CLEC-2 and its main ligand in hemostasis, leading to different studies trying to address this issue. Studies from May et al, using CLEC-2-deficient mouse platelets, demonstrated that this C-type lectin receptor is an essential mediator for platelet activation in vitro and in vivo, and that it is involved in aggregate and thrombus formation, and hence in hemostasis. ${ }^{115}$ However, another study using CLEC-2-deficient mice showed normal hemostasis in these animals. ${ }^{116}$ CLEC2 has also been implicated in thrombus stabilization, both in vitro and in vivo, despite the fact that bleeding tendency was not increased, contrary to previous data. ${ }^{117}$ Nevertheless, if there is a role for CLEC-2 in hemostasis, this cannot be attributed to podoplanin binding, since it is absent from the vascular injury site. ${ }^{87}$ The only circumstance in which podoplanin could be relevant in the thrombotic process is in advanced stages of atherosclerosis, since its expression was significantly enhanced in advanced atherosclerotic lesions, compared with early lesions. ${ }^{118}$ CLEC-2 actions in hemostasis itself, even if minor, would have to be triggered by ligands 
other than podoplanin, which have been already suggested before but not yet identified. ${ }^{117}$

CLEC-2 exists as a homodimer on the cell surface, which enables Syk's tandem SH2 domains to bind to the two phosphorylated hemITAM motifs of CLEC-2 homodimer, leading to tyrosine phosphorylation of downstream adapter proteins and effector enzymes, including PLC 2 , and consequently, platelets activation. ${ }^{119}$

Although CLEC-2 and the GPVI with its associated FCRrchains belong to the same family of ITAM receptors, and they trigger similar signaling events downstream of Syk, there is increasing evidence that the events in their signaling cascades are mechanistically distinct. ${ }^{70,87}$ The intracellular domain of CLEC- 2 contains a single YxxL motif, similar to GPVI. ${ }^{87}$ CLEC-2 signals through Src and Syk tyrosine kinases, leading to tyrosine phosphorylation and recruitment of adapter proteins, Tec family tyrosine kinases, and various effector proteins including PI 3-kinase, Vav, Rac1, and PLCY2. ${ }^{120}$

Platelet activation by rhodocytin, but not by collagen or by CVX, results in phosphorylation of the tyrosine residue in the YXXL motif. This phosphorylation depends on Syk and PLCY2, and partially on LAT, SLP-76, and Vav1/Vav3. ${ }^{111}$ The dependence on these signaling proteins differ between CLEC-2 and GPVI, since SLP-76 is an absolute requirement in GPVI signaling. ${ }^{121}$ Although CLEC-2 also translocates to lipid rafts upon ligand engagement, phosphorylation of CLEC-2 critically correlates with actin polymerization, Rac1 activation, and release of ADP and TXA $\mathrm{A}_{2}$, while the FcR $\gamma$-chain phosphorylation is independent of these requirements. ${ }^{122}$

A study by Bender et al targeted both GPVI and CLEC-2 simultaneously in mice with an antibody treatment, which led to defective hemostasis and impaired thrombus formation. The same was observed when GPVI and CLEC-2 were genetically inactivated in mice. ${ }^{123}$ These studies further revealed an unexpected functional redundancy of the two receptors in hemostasis and thrombosis. Moreover, they highlighted both receptors as promising targets for antithrombotic protection, which upon inactivation cause only moderately increased bleeding times in mice. ${ }^{123}$ These findings support the potential use of anti-GPVI and anti-CLEC-2based agents in the prevention of thrombotic diseases with patients at lower risk for hemorrhage.

\section{Current GPVI Inhibitors: Antibodies, GPVI-Fc Fusion Protein, and Bioactive Compounds from Plant Extracts and Snake Venom}

With the aim of decreasing thrombosis, clinical antiplatelet therapies have been developed, especially for the cohort of patients with higher tendency for cardiovascular events. A drawback of such antithrombotic therapies is the increased risk of bleeding. In several experimental models, blockage or depletion of GPVI not only impaired platelet activation and thrombus formation, but also did not result in major bleeding complications, thereby revealing striking benefits in treatment of thrombosis and thromboinflammation. ${ }^{12}$ This identified the role of GPVI as nonessential in hemostasis but nevertheless important in thrombosis, making its therapeu- tic targeting unlikely to lead to severe bleeding. Another advantage of targeting GPVI is the high specificity, since GPVI expression is restricted to platelets and megakaryocytes, which is important for minimizing potential side effects of a therapeutic agent. ${ }^{12}$ The available antiplatelet therapeutic agents and their targets in the different stages of platelet activation were listed by Andrews et al, in a review that can be consulted for more detailed information. ${ }^{12}$

In this section, we gathered the spectrum of various inhibitors that have been specifically developed to inhibit GPVI. The signaling pathways and the effect on platelet activation induced by GPVI-inhibition are summarized in - Table 3 .

One of the first described snake venom proteins that interacts with GPVI is CVX, isolated from the venom of Crotalus durissus. CVX is an agonist and activates platelets. ${ }^{151}$ Although numerous snake venom compounds have been described to activate platelets, recently, a peptide derived from a snake venom compound was shown to interact with GPVI, however, in an antagonistic way. The partial sequence of trowaglerix $\alpha$ subunit, a potent specific GPVI-targeting snake venom C-type lectin protein, isolated from the venom of Tropidolaemus wagleri, specifically inhibited collagen-induced human platelet aggregation. ${ }^{124}$ It is worth noting that there is a binding epitope on GPVI for polypeptides, such as hexa/decapeptides, which shows great potential for the development of lead compounds, aimed as small-mass GPVI antagonists for thrombogenic diseases. ${ }^{124}$ A mechanistically different snake venom metalloproteinase purified from the venom of Calloselasma rhodostoma, kistomin, inhibited collagen- and CVX-induced human platelet aggregation by cleavage of GPVI. ${ }^{125}$

A large number of natural bioactive compounds (NBCs), such as polyphenols, terpenoids, alkaloids, and fatty acids, were reported to have an apparent inhibitory activity on human platelets. ${ }^{152}$ However, their mode of action has remained elusive. Some of these compounds have been associated with GPVI and its signaling pathway. Buddleja globose, also known as matico, is a medicinal plant rich in polyphenols and flavonoids which present high antioxidant and anti-inflammatory activity. ${ }^{126}$ Matico inhibits human platelet aggregation response to collagen, CVX, and ADP in a concentration-dependent manner, as well as the expression of P-selectin, a maker of platelet activation. This antiplatelet activity most likely is caused by its high content in polyphenols. ${ }^{126}$ A potential mechanism of action for these compounds is through the inhibition of phosphorylation of PLC- $\gamma 2$ and PKC- $\beta 2$, which are signaling proteins downstream of GPVI. ${ }^{126}$ These findings have to be confirmed in clinical studies, to establish matico's suggested cardioprotective effects. Glaucocalyxin A, an ent-diterpenoid isolated and purified from the aerial parts of Rabdosia japonica var. glaucocalyx (Maxim.), disrupts CRP- and CVX-triggered human platelet activation in addition to thrombus formation in mice, also through the inhibition of collagen-stimulated tyrosine phosphorylation of Syk, LAT, and PLCY2. ${ }^{127}$ Fractions of aqueous and methanolic extracts from Solanum lycopersicum, the well-known tomato, also possesses antiaggregating activity as they inhibit collagen-induced rat platelet aggregation, potentially via GPVI receptor. ${ }^{128}$ 


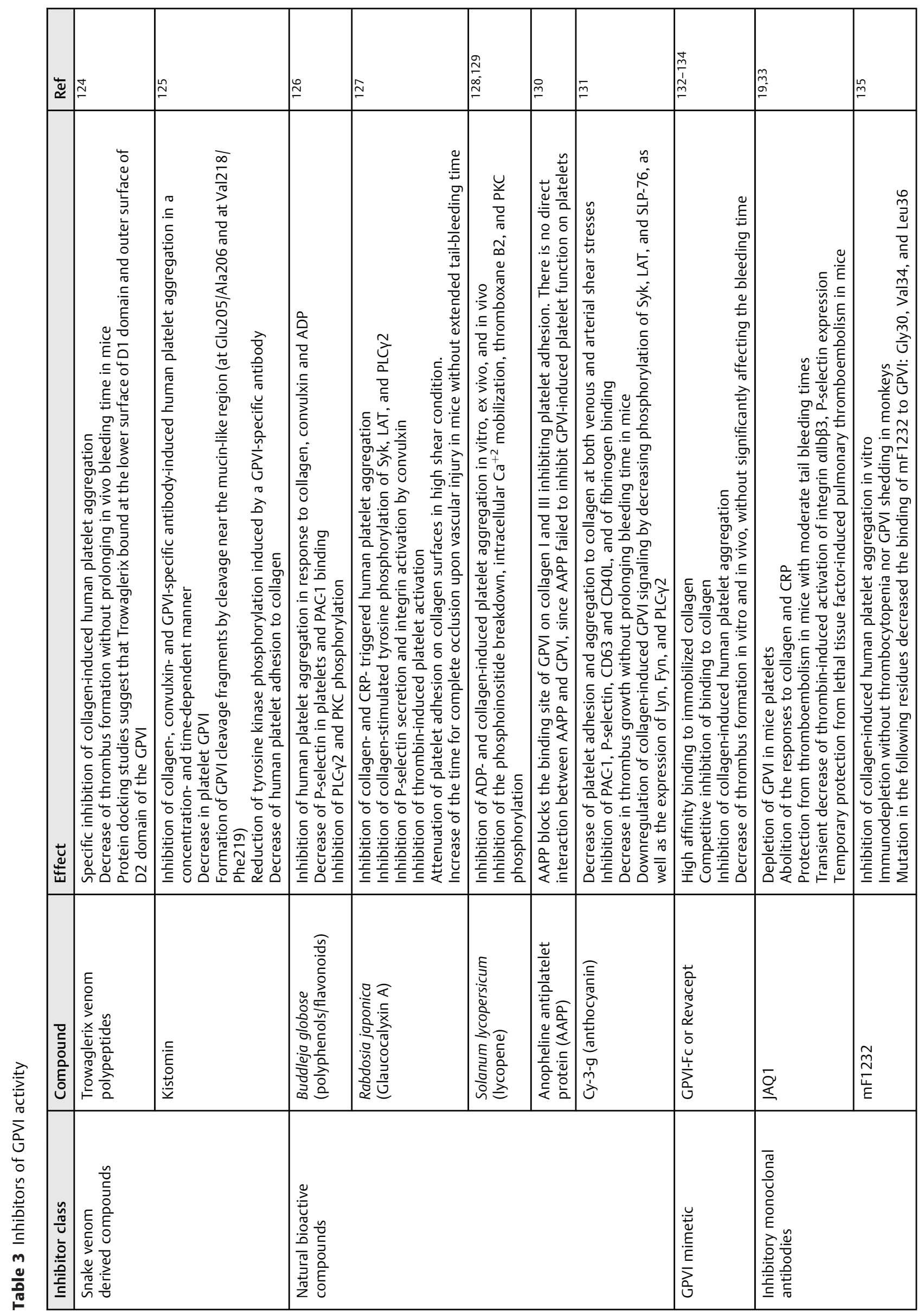




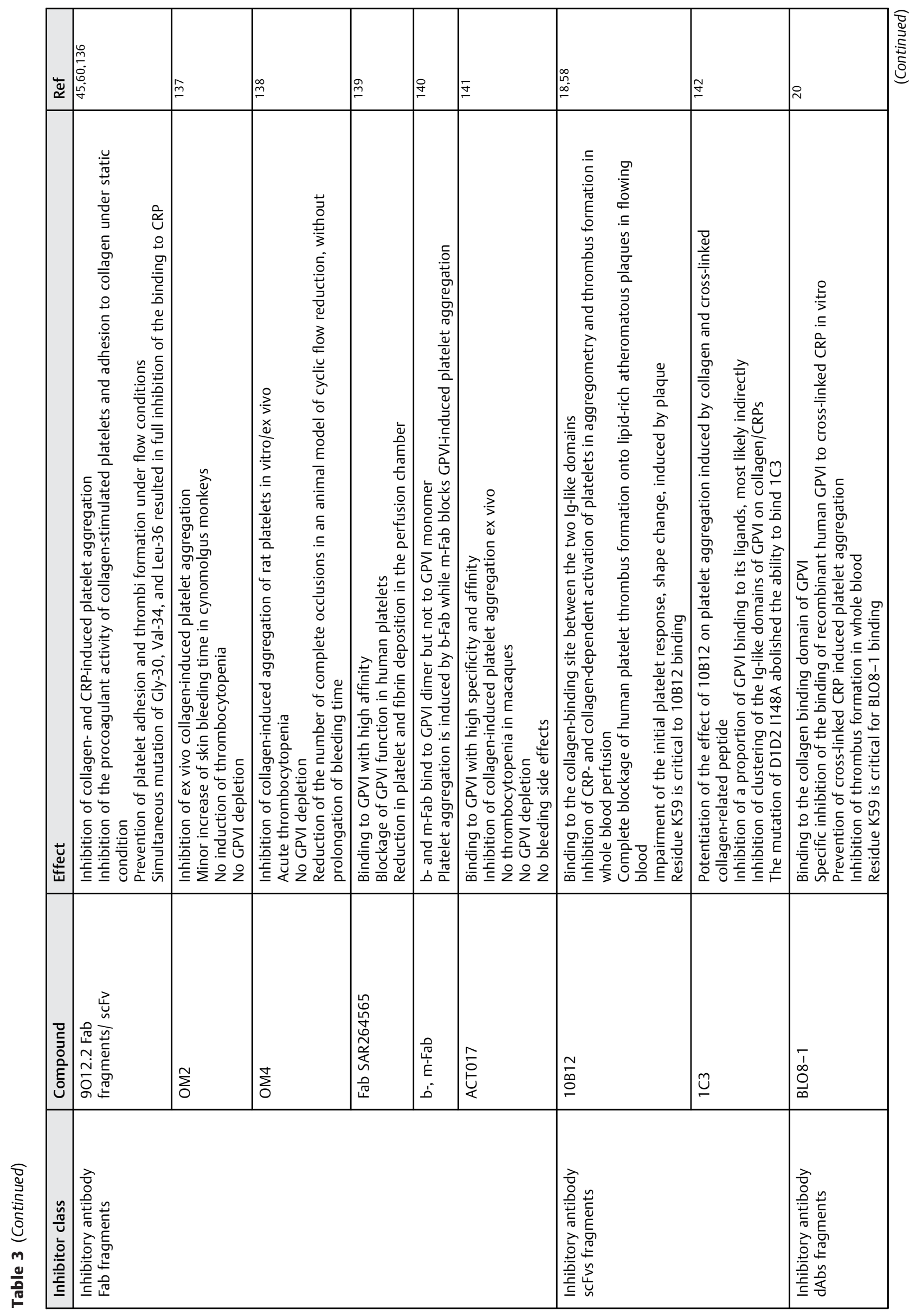




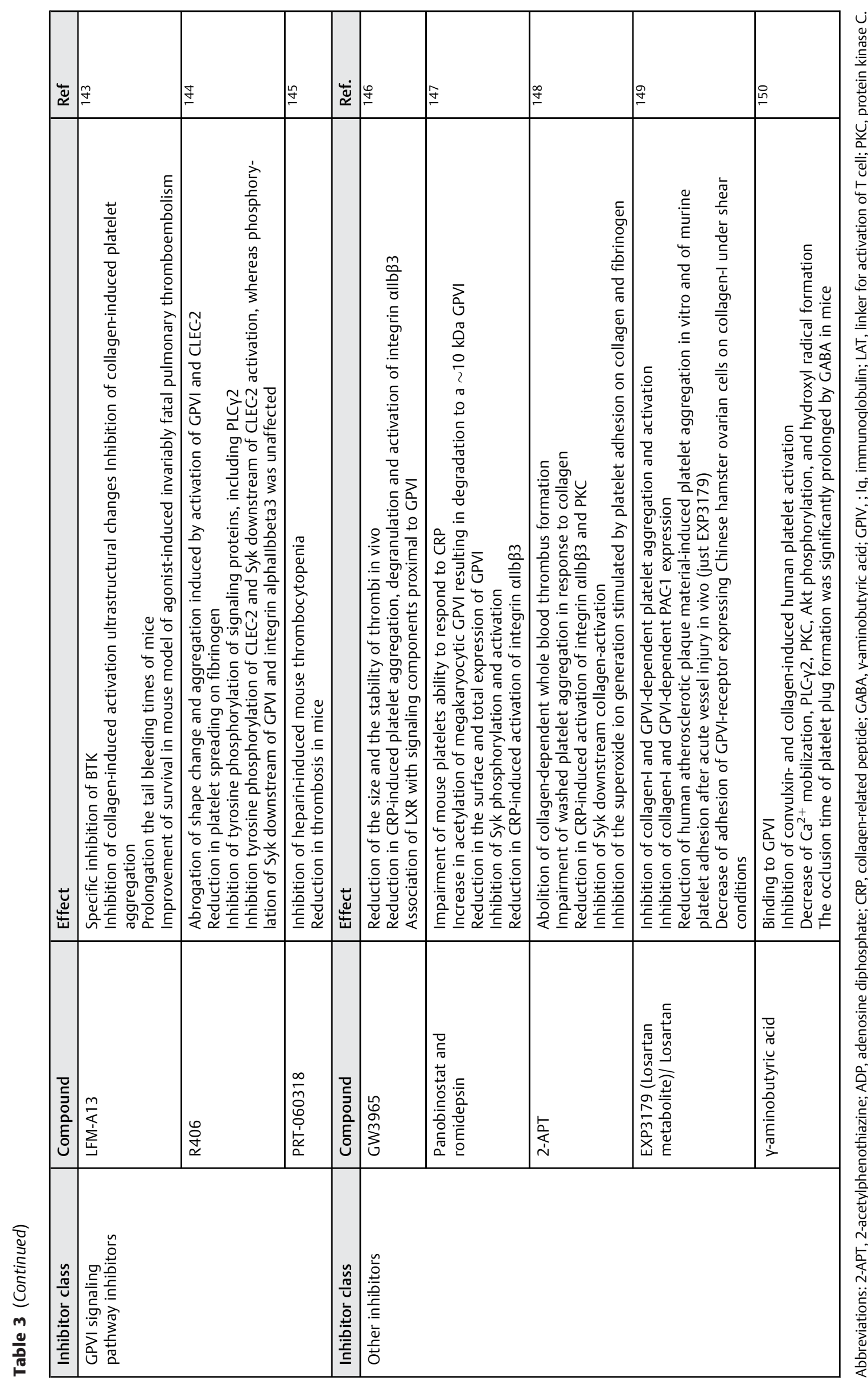


Furthermore, another publication describes lycopene, a natural carotenoid antioxidant presents in tomatoes, as an active compound with in vitro and in vivo antiplatelet activity, concentration-dependently inhibiting the breakdown of phosphoinositide, intracellular $\mathrm{Ca}^{+2}$ mobilization, TXB2 formation upon stimulation by collagen, and PKC phosphorylation. $^{129}$

Interestingly, the inhibitory effect of NBCs on platelets goes beyond the direct interaction with GPVI. As reported by Yoshida et al, anopheline antiplatelet protein (AAPP), a saliva protein from malaria vector mosquito, inhibits human platelet adhesion by blocking the binding sites of GPVI on collagen. Interestingly, AAPP has no direct effect on GPVI-induced platelet activation, and therefore, it could be used as a tool to indirectly inhibit the GPVI-induced platelet functions with the benefits of potential minor side effects. ${ }^{130}$

Another potential NBCs mechanism of action involves peroxisome proliferator-activated receptor (PPAR) and the inhibition of protein kinase $A$ and $G$, cyclooxygenase-1, TXA , and cytosolic $\mathrm{Ca}^{2+}$. An interesting study by Akbiyik et al associated PPAR- $\gamma$ of platelets with GPVI signaling. ${ }^{153}$ By treating collagen-stimulated platelets with PPAR- $\gamma$ ligands, such as 15d-PGJ2 and rosiglitazone, the authors demonstrated that these ligands modulate the activity of the GPVI signaling pathway, resulting in a decrease of human platelet activation, aggregation, and thrombus formation under arterial flow conditions. ${ }^{154}$ Cyanidin 3-O- $\beta$-glucopyranoside, an anthocyanin from the flavonoid family, attenuated thrombus growth without prolonging bleeding time in mice, downregulated collagen-induced GPVI signaling by significantly decreasing phosphorylation of Syk, LAT, and SLP-76, as well as the expression of Lyn, Fyn, and PLC- $\gamma 2 .^{131}$

To date, the main clinical strategies developed focus on interfering with the GPVI-collagen interaction by two main approaches: using collagen-binding molecules or through GPVI-function blocking reagents.

A GPVI mimetic has been developed, a recombinant fusion protein that consists of the extracellular collagen-binding domain of GPVI fused with the C-terminal of human Ig Fc domain, forming a soluble GPVI dimeric called GPVI-Fc or Revacept. ${ }^{132}$ This Fc fusion protein specifically binds to immobilized collagen, with high affinity, and, as a decoy receptor, it competitively inhibits binding of collagen to platelet-anchored human GPVI. ${ }^{132}$ Furthermore, in response to Revacept, thrombus formation is attenuated in vitro when platelets are under shear conditions. In an in vivo setting, the platelet-vessel wall interactions are compromised by abolishing stable arrest and platelet aggregation following vascular injury. ${ }^{132,133}$ Most importantly, when delivered locally at the site of vascular injury, the amount of GPVI-Fc is sufficient to reduce thrombus formation without systemic antiplatelet effects, as shown in a rabbit model. ${ }^{133}$ In a clinical phase I study, Revacept did not significantly affect bleeding time, while collagen-induced platelet aggregation was dose-dependently inhibited. ${ }^{134}$ Some authors have assessed the combined administration of Revacept with first line treatments, as an alternative thrombolytic therapy. The main goal of the work from Reimann et al was to establish a thrombolytic therapy regimen using a mouse model of acute ischemic stroke, with concomitant administration of the first line treatment, recombinant tissue plasminogen activator (rtPA) and Revacept. ${ }^{155}$ By combining lower doses of rtPA with Revacept, treatment efficacy improved without increased risk of intracerebral hemorrhage, while higher doses of rtPA sustained a significant risk of bleeding risk. ${ }^{155}$ The overall results of Revacept characterize this compound as a safe and reliable alternative for antiplatelet therapy. A phase II study has taken place to assess the detailed clinical settings and pathologies where this therapy could carry advantageous outcomes (NCT01645306).

Among the approaches of directly targeting GPVI, inhibiting antibodies have been developed. The first monoclonal antibody ( $\mathrm{mAb}$ ) against GPVI was described by Nieswandt et al, as JAQ1. It inhibited mouse platelet aggregation induced by collagen, however, when cross-linked, this antibody had the opposite effect. ${ }^{156}$ This is a disadvantage of the monoclonal antibodies, as the Fc domain can cross-link and cluster the GPVI. ${ }^{12}$ Nevertheless, upon JAQ1 treatment, GPVI was depleted from circulating platelets in mice and the thrombotic responses to collagen and CRPs were abolished, resulting in protection from thromboembolism with moderate tail bleeding times. ${ }^{19,33}$ Other mAbs, the human GPVI-specific mouse mF1201 and mF1232, were also assessed in in vitro studies with different effects. The antibody mF1201 induced human platelet activation and GPVI shedding, while mF1232 inhibited collagen-induced human platelet aggregation. ${ }^{135}$ This was reflected in studies in vivo, where monkeys treated with mF1232 suffered from long-term antiplatelet effects due to GPVI shedding-dependent immunodepletion without significant thrombocytopenia. ${ }^{135}$

To overcome the potential undesired platelet activation triggered by mAbs, inhibitory Fab fragments were developed. GPVI-blocking antibody fragments, such as antigen-binding Fab or $\mathrm{F}\left(\mathrm{ab}^{\prime}\right)_{2}$ fragments, are generated from monoclonal anti-GPVI antibodies by limited proteolysis with soluble papain or immobilized pepsin, respectively. ${ }^{45}$ Interestingly, the development of these fragments was based on the pathological case of a patient suffering from purpura and prolonged bleeding time despite an adequate number of platelets. ${ }^{45}$ The $F\left(\mathrm{ab}^{\prime}\right)_{2}$ fragments of antibodies isolated from the patient's blood induced TXB2 synthesis, increased cytoplasmic $\mathrm{Ca}^{2+}$ levels in platelets and specifically inhibited aggregation induced by collagen. ${ }^{21}$ In line with these findings, antagonistic Fab fragments generated from the $\mathrm{mAb}$ 9012.2 have been developed. This mAb was selected because, although 9012.2 IgGs induced platelet activation, it also disrupted the interaction of GPVI with collagen. ${ }^{45} 9012.2$ Fab fragments inhibit collagen- and CRP-induced platelet aggregation and prevent thrombus formation under flow conditions. ${ }^{45}$ In addition, a successful screening has identified Fab fragments that specifically bind to GPVI dimer, but do not bind to GPVI monomer. ${ }^{140}$ The promising results spurred the design and humanization of the single-chain variable fragment (scFv) from the $9012 \mathrm{mAb}$ by recombinant protein engineering. By preserving the functional properties, these Fab fragments can potentially be exploited for 
therapeutic applications. ${ }^{136}$ For example, ACT017 is a high affinity humanized Fab that inhibits collagen-induced human platelet aggregation, and showed no thrombocytopenia, GPVI depletion, or bleeding side effects in macaques ${ }^{141}$ and is currently being tested in phase II studies (NCT03803007). The inhibition of platelet function by fragments (Fab, scFvs, and $\mathrm{dAbs}$ ) generated from other antibodies was also later reported and their effects are summarized in the - Table 3. ${ }^{18,20,58,137-139,142}$

Not included in any of the named categories, additional inhibitors to GPVI have been described. Compounds targeting tyrosine kinases and adaptor proteins downstream of GPVI also block the signaling pathway and function of GPVI. One of the first studies describing an inhibitor of the GPVI pathway was a leflunomide metabolite analog, LFM-A13, and it was designed to specifically bind to one of the TEC family protein tyrosine kinase, Btk. ${ }^{143}$ Later, two other Syk inhibitory molecules, R406 ${ }^{144}$ and PRT-060318, ${ }^{145}$ emerged as tools to study the GPVI and CLEC-2 signaling pathways. All compounds inhibit platelet activation. R406 decreases the tyrosine phosphorylation of signaling proteins, such as PLC$\checkmark 2$, in human platelets and PRT-060318 reduces heparininduced thrombocytopenia and thrombosis in mice. ${ }^{143-145}$
Other compounds also interfere with the GPVI signaling pathway but not by targeting signalosome molecules. An example is GW3965, a transcription factors ligand involved in the regulation of cholesterol homeostasis and liver $\mathrm{X}$ receptors, which is expressed in platelets. ${ }^{146}$ Spyridon et al, attributed the atheroprotective properties of GW3965 to the antiplatelet and antithrombotic effects, since it nongenomically modulated human platelet aggregation and reduced the size and stability of thrombi in an in vivo mouse model. ${ }^{146}$ These effects are suggested to be mechanistically related to the GPVI signaling pathway. ${ }^{146}$ Inhibitors of the enzyme histone deacetylase, panobinostat, and romidepsin, although structurally different, both impaired mouse platelets ability to respond to CRP, increased acetylation of megakaryocytic GPVI resulting in degradation to approximately $10 \mathrm{kDa}$ GPVI and inhibited Syk phosphorylation and activation. ${ }^{147}$ Interestingly, NADPH oxidases (NOXs) are involved in platelet activation (mechanism remains largely unknown) and was elucidated by the NOX inhibitor 2acetylphenothiazine (2-APT). ${ }^{148}$ 2-APT also abolished the collagen-dependent formation of whole blood thrombus and aggregation of washed human platelets. Moreover, the CRPtriggered activation of integrin $\alpha$ IIb $\beta 3$, PKC, and collagen-

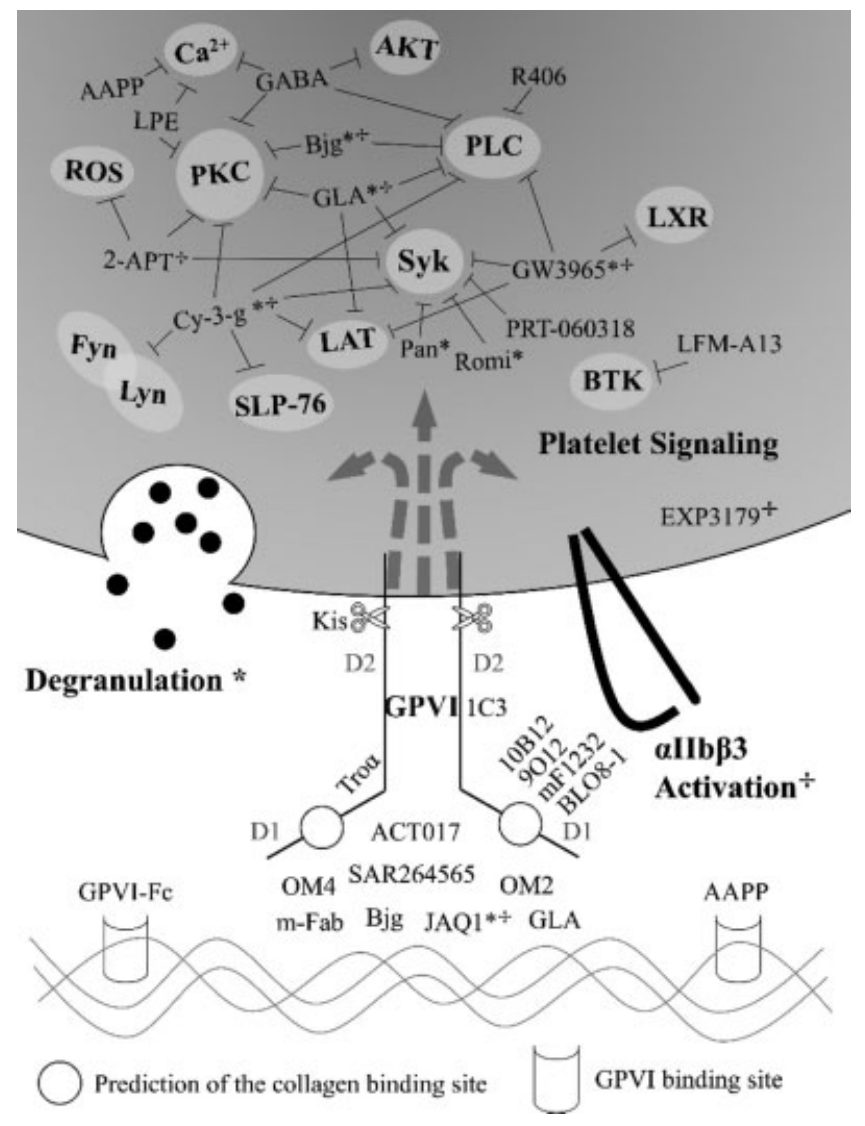

Fig. 2 Inhibitors of glycoprotein (GP) VI activity. Snake venom derived compounds: Trowaglerix venom polypeptides (Troa10); Kistomin (Kis); Natural bioactive compounds: Buddleja globose (Bjg); Rabdosia japonica - Glaucocalyxin A (GLA); Solanum lycopersicum (lycopene) - LPE; Anopheline antiplatelet protein (AAPP); Anthocyanin (cyanidin-3-glucoside) - Cy-3-g; GPVI mimetic: GPVI-Fc (Revacept); Inhibitory monoclonal antibodies: JAQ1; mF1232; Inhibitory antibody Fab fragments: 9012.2; OM2; OM4; SAR264565; m-Fab; ACT017; Inhibitory antibody scFvs fragments: 10B12; 1C3; Inhibitory antibody dAbs fragments: BLO8-1; GPVI signaling pathway inhibitors: LFM-A13; R406; PRT-060318; Other inhibitors: GW3965; Panobinostat - Pan; romidepsin - Romi; 2-APT; EXP3179 (Losartan metabolite); $\gamma$-aminobutyric acid - GABA. * GPVI-induced degranulation inhibition; inhibition of GPVI-induced $\alpha$ llb $\beta 3$ activation. 
activation of Syk was inhibited by 2 -APT. ${ }^{148}$ The signaling molecules of the GPVI pathway are an appealing target for therapy, but the lack of specificity poses a significant challenge, as Syk and other components of the GPVI signalosome are also important in immune cell signal transduction. ${ }^{157}$ Another potential concern of this targeting is the effect of disrupting GPVI receptor signaling via the ITAM signaling receptors on platelets, which could also negatively affect the outside-in signaling of the $\alpha$ IIb $\beta 3$ and $\beta 1$ integrins. Nevertheless, early studies using bone marrow chimeric Syk-deficient mice or pharmacological inhibition of Syk in vivo reported virtually normal hemostasis. Therefore, targeting GPVI-mediated production of reactive oxygen species or the transcription factors may present more subtle effects by diminishing but not blocking GPVI-signaling function. ${ }^{12,157}$

More blocking inhibitors of GPVI have also been found in an intuitively less expected manner. EXP3179 is an active metabolite that arises from the angiotensin II type 1 (AT1)-receptor antagonist losartan (LOS) after hepatic metabolization by the cytochrome-P450 pathway. Both EXP3179 and LOS, inhibit collagen I- and GPVI-dependent human platelet aggregation and activation. ${ }^{149}$ EXP3179 reduces platelet aggregation induced by human atherosclerotic plaque material in vitro, as well as murine platelet adhesion after acute vessel injury in vivo. ${ }^{149}$ The endogenously produced molecule, $\gamma$-aminobutyric acid (GABA), also showed a direct inhibitory effect on GPVI. GABA is the major inhibitory neurotransmitter in the central nervous system, and specifically inhibits collagen-induced platelet activation, by binding to GPVI, resulting in decreased $\mathrm{Ca}^{2+}$ mobilization, PLC- $\gamma 2$, PKC, Akt phosphorylation, and hydroxyl radical formation. ${ }^{150}$

Recently, our group has initiated drug discovery attempts to identify new small molecules capable of inhibiting the activity of GPVI. For that, a 384-well plate assay to test platelet function in a high-throughput screening format was developed and, using compounds selected by structure-based virtual screening, we identified several hits that markedly decreased the human platelet activation induced by CRP. ${ }^{158}$ Currently, additional experiments are being performed to test the specificity of these hit compounds and their effect on thrombus formation.

\section{Conclusion}

This review summarizes several reports demonstrating the importance of GPVI receptor on hemostasis, platelet function, and thrombus formation by means of human GPVI-deficiency studies. In addition, the recent discoveries related to the biochemical structure, intracellular signaling, and potential inhibitors of GPVI receptor were included in this review. Due to its characteristic features, GPVI is crucial for thrombus formation and is a potential drug target for thrombosis. The targets of the different inhibitors on platelet GPVI, as well as downstream signaling pathways are illustrated in -Fig. 2.

Differently from human deficiency of $\alpha 2 \beta 1$ integrin, mutations of GPVI receptor in patients lead, at most, to mild bleeding. In addition, the role of GPVI in platelet function seems to induce a rapid activation of $\alpha$ IIb $\beta 3$ integrin and platelet degranulation, crucial steps for aggregation formation. Therefore, GPVI is an appealing therapeutic target, and parallel efforts have led to the development of several compounds that are able to inhibit GPVI activity. The insights that these studies provided and the early stage of development of an ideal antiplatelet agent to target GPVI, have prompted further research aimed at developing a new GPVI-inhibiting drug. In addition, the "bleeding-free" feature of GPVI inhibitors ensures a clinical advantage compared with all antiplatelet drugs available on the market. Taken together, the purpose of this review was to provide guidance for future investigation in the platelet field, specifically for GPVI-related research, and potentially help with the development of a novel drug class for antithrombotic therapy.

\section{Funding}

A.C.M.C. was funded by EU under the People Program (Marie Curie Actions) of the European Union's Seventh Framework Program FP7/2007 -2013/ under the REA grant agreement $\mathrm{n}^{\circ}$ (316610).

\section{Conflict of Interest}

None declared.

\section{References}

1 World Health Organization. Disease Burden and Mortality Estimates: Disease Burden 2000-2016; 2016. Available at: http://www.who.int/healthinfo/global_burden_disease/estima tes/en/. Accessed August 7, 2019

2 Michelson AD. Antiplatelet therapies for the treatment of cardiovascular disease. Nat Rev Drug Discov 2010;9(02):154-169

3 Hansson GK. Inflammatory mechanisms in atherosclerosis. J Thromb Haemost 2009;7(Suppl 1):328-331

4 Koupenova M, Kehrel BE, Corkrey HA, Freedman JE. Thrombosis and platelets: an update. Eur Heart J 2017;38(11):785-791

5 Collaboration AT. Collaborative overview of randomised trials of antiplatelet therapy Prevention of death, myocardial infarction, and stroke by prolonged antiplatelet therapy in various categories of patients. BMJ 1994;308(6921):81-106

6 Collaboration AT; Antithrombotic Trialists' Collaboration. Collaborative meta-analysis of randomised trials of antiplatelet therapy for prevention of death, myocardial infarction, and stroke in high risk patients. BMJ 2002;324(7329):71-86

7 Investigators E; EPIC Investigators. Use of a monoclonal antibody directed against the platelet glycoprotein IIb/IIIa receptor in highrisk coronary angioplasty. N Engl J Med 1994;330(14):956-961

8 Serebruany VL, Malinin AI, Ferguson JJ, Vahabi J, Atar D, Hennekens $\mathrm{CH}$. Bleeding risks of combination vs. single antiplatelet therapy: a meta-analysis of 18 randomized trials comprising 129,314 patients. Fundam Clin Pharmacol 2008;22(03):315-321

9 Hansen ML, Sørensen R, Clausen MT, et al. Risk of bleeding with single, dual, or triple therapy with warfarin, aspirin, and clopidogrel in patients with atrial fibrillation. Arch Intern Med 2010; 170(16):1433-1441

10 Li L, Geraghty OC, Mehta Z, Rothwell PM; Oxford Vascular Study. Age-specific risks, severity, time course, and outcome of bleeding on long-term antiplatelet treatment after vascular events: a population-based cohort study. Lancet 2017;390(10093):490-499

11 Jiang P, Jandrot-Perrus M. New advances in treating thrombotic diseases: GPVI as a platelet drug target. Drug Discov Today 2014; 19(09):1471-1475

12 Andrews RK, Arthur JF, Gardiner EE. Targeting GPVI as a novel antithrombotic strategy. J Blood Med 2014;5:59-68 
13 Dumont B, Lasne D, Rothschild C, et al. Absence of collageninduced platelet activation caused by compound heterozygous GPVI mutations. Blood 2009;114(09):1900-1903

14 Bigalke B, Stellos K, Geisler T, et al. Expression of platelet glycoprotein VI is associated with transient ischemic attack and stroke. Eur J Neurol 2010;17(01):111-117

15 Bigalke B, Stellos K, Stakos D, et al. Influence of platelet count on the expression of platelet collagen receptor glycoprotein VI (GPVI) in patients with acute coronary syndrome. Thromb Haemost 2009;101(05):911-915

16 Vélez P, Ocaranza-Sánchez R, López-Otero D, et al. Alteration of platelet GPVI signaling in ST-elevation myocardial infarction patients demonstrated by a combination of proteomic, biochemical, and functional approaches. Sci Rep 2016;6:39603

17 Kato K, Kanaji T, Russell S, et al. The contribution of glycoprotein VI to stable platelet adhesion and thrombus formation illustrated by targeted gene deletion. Blood 2003;102(05):1701-1707

18 Penz S, Reininger AJ, Brandl R, et al. Human atheromatous plaques stimulate thrombus formation by activating platelet glycoprotein VI. FASEB J 2005;19(08):898-909

19 Schulte V, Reusch HP, Pozgajová M, Varga-Szabó D, Gachet C, Nieswandt B. Two-phase antithrombotic protection after antiglycoprotein VI treatment in mice. Arterioscler Thromb Vasc Biol 2006;26(07):1640-1647

20 Walker A, Pugh N, Garner SF, et al; Bloodomics Consortium. Single domain antibodies against the collagen signalling receptor glycoprotein VI are inhibitors of collagen induced thrombus formation. Platelets 2009;20(04):268-276

21 Sugiyama T, Okuma M, Ushikubi F, Sensaki S, Kanaji K, Uchino H. A novel platelet aggregating factor found in a patient with defective collagen-induced platelet aggregation and autoimmune thrombocytopenia. Blood 1987;69(06):1712-1720

22 Kojima H, Moroi M, Jung SM, et al. Characterization of a patient with glycoprotein (GP) VI deficiency possessing neither antiGPVI autoantibody nor genetic aberration. JThromb Haemost 2006;4(11):2433-2442

23 Moroi M, Jung SM, Okuma M, Shinmyozu K. A patient with platelets deficient in glycoprotein VI that lack both collageninduced aggregation and adhesion. JClin Invest 1989;84(05): 1440-1445

24 Arai M, Yamamoto N, Moroi M, Akamatsu N, Fukutake K, Tanoue K. Platelets with $10 \%$ of the normal amount of glycoprotein VI have an impaired response to collagen that results in a mild bleeding tendency. Br J Haematol 1995;89(01):124-130

25 Hermans C, Wittevrongel C, Thys C, Smethurst PA, Van Geet C, Freson K. A compound heterozygous mutation in glycoprotein $\mathrm{VI}$ in a patient with a bleeding disorder. JThromb Haemost 2009;7 (08):1356-1363

26 Ryo R, Yoshida A, Sugano W, et al. Deficiency of P62, a putative collagen receptor, in platelets from a patient with defective collagen-induced platelet aggregation. Am J Hematol 1992;39 (01):25-31

27 Takahashi H, Moroi M. Antibody against platelet membrane glycoprotein VI in a patient with systemic lupus erythematosus. Am J Hematol 2001;67(04):262-267

28 Boylan B, Chen H, Rathore V, et al. Anti-GPVI-associated ITP: an acquired platelet disorder caused by autoantibody-mediated clearance of the GPVI/FcRgamma-chain complex from the human platelet surface. Blood 2004;104(05):1350-1355

29 Nurden P, Jandrot-Perrus M, Combrié R, et al. Severe deficiency of glycoprotein VI in a patient with gray platelet syndrome. Blood 2004;104(01):107-114

30 Gardiner EE, Al-Tamimi M, Mu F-T, et al. Compromised ITAMbased platelet receptor function in a patient with immune thrombocytopenic purpura. JThromb Haemost 2008;6(07): $1175-1182$

31 Akiyama M, Kashiwagi H, Todo K, et al. Presence of plateletassociated anti-glycoprotein (GP)VI autoantibodies and restora- tion of GPVI expression in patients with GPVI deficiency. J Thromb Haemost 2009;7(08):1373-1383

32 Matus V, Valenzuela G, Sáez CG, et al. An adenine insertion in exon 6 of human GP6 generates a truncated protein associated with a bleeding disorder in four Chilean families. JThromb Haemost 2013;11(09):1751-1759

33 Nieswandt B, Schulte V, Bergmeier W, et al. Long-term antithrombotic protection by in vivo depletion of platelet glycoprotein VI in mice. JExp Med 2001;193(04):459-469

34 Boilard E, Nigrovic PA, Larabee K, et al. Platelets amplify inflammation in arthritis via collagen-dependent microparticle production. Science 2010;327(5965):580-583

35 Jain S, Russell S, Ware J. Platelet glycoprotein VI facilitates experimental lung metastasis in syngenic mouse models. JThromb Haemost 2009;7(10):1713-1717

36 Berlanga O, Bobe R, Becker M, et al. Expression of the collagen receptor glycoprotein VI during megakaryocyte differentiation. Blood 2000;96(08):2740-2745

37 Ichinohe T, Takayama H, Ezumi Y, Yanagi S, Yamamura H, Okuma M. Cyclic AMP-insensitive activation of c-Src and Syk protein-tyrosine kinases through platelet membrane glycoprotein VI. JBiol Chem 1995;270(47):28029-28036

38 Ichinohe T, Takayama H, Ezumi Y, et al. Collagen-stimulated activation of Syk but not c-Src is severely compromised in human platelets lacking membrane glycoprotein VI. J Biol Chem 1997; 272(01):63-68

39 Kehrel B, Wierwille S, Clemetson KJ, et al. Glycoprotein VI is a major collagen receptor for platelet activation: it recognizes the platelet-activating quaternary structure of collagen, whereas CD36, glycoprotein IIb/IIIa, and von Willebrand factor do not. Blood 1998;91(02):491-499

40 Sugiyama T, Ishibashi T, Okuma M. Functional role of the antigen recognized by an antiplatelet antibody specific for a putative collagen receptor in platelet-collagen interaction. Int J Hematol 1993;58(1-2):99-104

41 Tsuji M, Ezumi Y, Arai M, Takayama H. A novel association of Fc receptor gamma-chain with glycoprotein VI and their co-expression as a collagen receptor in human platelets. J Biol Chem 1997;272(38):23528-23531

42 Moroi M, Jung SM, Shinmyozu K, Tomiyama Y, Ordinas A, DiazRicart M. Analysis of platelet adhesion to a collagen-coated surface under flow conditions: the involvement of glycoprotein VI in the platelet adhesion. Blood 1996;88(06):2081-2092

43 Qiao J, Al-Tamimi M, Baker RI, Andrews RK, Gardiner EE. The platelet Fc receptor, FcrRIla. Immunol Rev 2015;268(01):241-252

44 Matsumoto Y, Takizawa H, Gong X, et al. Highly potent antihuman GPVI monoclonal antibodies derived from GPVI knockout mouse immunization. Thromb Res 2007;119(03):319-329

45 Lecut C, Feeney LA, Kingsbury G, et al. Human platelet glycoprotein VI function is antagonized by monoclonal antibody-derived Fab fragments. JThromb Haemost 2003;1(12):2653-2662

46 Onselaer M-B, Hardy AT, Wilson C, et al. Fibrin and D-dimer bind to monomeric GPVI. Blood Adv 2017;1(19):1495-1504

47 Lima AM, Wegner SV, Martins Cavaco AC, et al. The spatial molecular pattern of integrin recognition sites and their immobilization to colloidal nanobeads determine $\alpha 2 \beta 1$ integrin-dependent platelet activation. Biomaterials 2018;167:107-120

48 Nieuwenhuis HK, Akkerman JWN, Houdijk WPM, Sixma JJ. Human blood platelets showing no response to collagen fail to express surface glycoprotein Ia. Nature 1985;318(6045):470-472

49 Nieuwenhuis HK, Sakariassen KS, Houdijk WP, Nievelstein PF, Sixma JJ. Deficiency of platelet membrane glycoprotein Ia associated with a decreased platelet adhesion to subendothelium: a defect in platelet spreading. Blood 1986;68(03):692-695

50 Kehrel B, Balleisen L, Kokott R, et al. Deficiency of intact thrombospondin and membrane glycoprotein la in platelets with defective collagen-induced aggregation and spontaneous loss of disorder. Blood 1988;71(04):1074-1078 
51 Handa M, Watanabe K, Kawai Y, et al. Platelet unresponsiveness to collagen: involvement of glycoprotein Ia-IIa (alpha 2 beta 1 integrin) deficiency associated with a myeloproliferative disorder. Thromb Haemost 1995;73(03):521-528

52 Moroi M, Jung SM. Platelet glycoprotein VI: its structure and function. Thromb Res 2004;114(04):221-233

53 Ezumi Y, Uchiyama T, Takayama H. Molecular cloning, genomic structure, chromosomal localization, and alternative splice forms of the platelet collagen receptor glycoprotein VI. Biochem Biophys Res Commun 2000;277(01):27-36

54 Pollitt AY, Hughes CE, Watson SP. GPVI and CLEC-2. In: Platelets. Elsevier; 2013:215-231. Available at: http://linkinghub.elsevier. com/retrieve/pii/B9780123878373000110. Accessed August 7, 2019

55 Bori-Sanz T, Inoue KS, Berndt MC, Watson SP, Tulasne D. Delineation of the region in the glycoprotein VI tail required for association with the Fc receptor gamma-chain. JBiol Chem 2003;278(38):35914-35922

56 Andrews RK, Suzuki-Inoue K, Shen Y, Tulasne D, Watson SP, Berndt MC. Interaction of calmodulin with the cytoplasmic domain of platelet glycoprotein VI. Blood 2002;99(11):4219-4221

57 Suzuki-Inoue K, Tulasne D, Shen Y, et al. Association of Fyn and Lyn with the proline-rich domain of glycoprotein VI regulates intracellular signaling. J Biol Chem 2002;277(24):21561-21566

58 Smethurst PA, Joutsi-Korhonen L, O'Connor MN, et al. Identification of the primary collagen-binding surface on human glycoprotein VI by site-directed mutagenesis and by a blocking phage antibody. Blood 2004;103(03):903-911

59 O'Connor MN, Smethurst PA, Farndale RW, Ouwehand WH. Gainand loss-of-function mutants confirm the importance of apical residues to the primary interaction of human glycoprotein VI with collagen. J Thromb Haemost 2006;4(04):869-873

60 Lecut $\mathrm{C}$, Arocas V, Ulrichts $\mathrm{H}$, et al. Identification of residues within human glycoprotein VI involved in the binding to collagen: evidence for the existence of distinct binding sites. JBiol Chem 2004;279(50):52293-52299

61 Kunicki TJ, Cheli Y, Moroi M, Furihata K. The influence of Nlinked glycosylation on the function of platelet glycoprotein VI. Blood 2005;106(08):2744-2749

62 Arthur JF, Shen Y, Kahn ML, Berndt MC, Andrews RK, Gardiner EE. Ligand binding rapidly induces disulfide-dependent dimerization of glycoprotein VI on the platelet plasma membrane. J Biol Chem 2007;282(42):30434-30441

63 Horii K, Kahn ML, Herr AB. Structural basis for platelet collagen responses by the immune-type receptor glycoprotein VI. Blood 2006;108(03):936-942

64 Feitsma LJ, Brondijk THC, Jarvis G, et al. Structural insights into collagen-binding by platelet receptor Glycoprotein VI. Doi: 10.2210/pdb50U7/pdb

65 Gibbins JM, Okuma M, Farndale R, Barnes M, Watson SP. Glycoprotein VI is the collagen receptor in platelets which underlies tyrosine phosphorylation of the Fc receptor $\gamma$-chain. FEBS Lett 1997;413(02):255-259

66 Berlanga O, Tulasne D, Bori T, et al. The Fc receptor gamma-chain is necessary and sufficient to initiate signalling through glycoprotein VI in transfected cells by the snake C-type lectin, convulxin. Eur J Biochem 2002;269(12):2951-2960

67 Berlanga O, Bori-Sanz T, James JR, et al. Glycoprotein VI oligomerization in cell lines and platelets. J Thromb Haemost 2007;5 (05):1026-1033

68 Miura Y, Takahashi T, Jung SM, Moroi M. Analysis of the interaction of platelet collagen receptor glycoprotein VI (GPVI) with collagen. A dimeric form of GPVI, but not the monomeric form, shows affinity to fibrous collagen. JBiol Chem 2002;277 (48):46197-46204

69 Plenz GAM, Deng MC, Robenek H, Völker W. Vascular collagens: spotlight on the role of type VIII collagen in atherogenesis. Atherosclerosis 2003;166(01):1-11
70 Watson SP, Herbert JMJ, Pollitt AY. GPVI and CLEC-2 in hemostasis and vascular integrity. JThromb Haemost 2010;8(07): 1456-1467

71 Jung SM, Takemura Y, Imamura Y, Hayashi T, Adachi E, Moroi M. Collagen-type specificity of glycoprotein VI as a determinant of platelet adhesion. Platelets 2008;19(01):32-42

72 Mizuno K, Adachi E, Imamura Y, Katsumata O, Hayashi T. The fibril structure of type $\mathrm{V}$ collagen triple-helical domain. Micron $2001 ; 32(03): 317-323$

73 Jarvis GE, Atkinson BT, Snell DC, Watson SP. Distinct roles of GPVI and integrin alpha(2)beta(1) in platelet shape change and aggregation induced by different collagens. Br J Pharmacol 2002; 137(01):107-117

74 Hechler B, Nonne C, Eckly A, et al. Arterial thrombosis: relevance of a model with two levels of severity assessed by histologic, ultrastructural and functional characterization. JThromb Haemost 2010;8(01):173-184

75 Knight CG, Morton LF, Onley DJ, et al. Collagen-platelet interaction: Gly-Pro-Hyp is uniquely specific for platelet Gp VI and mediates platelet activation by collagen. Cardiovasc Res 1999;41 (02):450-457

76 Nieswandt B, Watson SP. Platelet-collagen interaction: is GPVI the central receptor? Blood 2003;102(02):449-461

77 Morton LF, Hargreaves PG, Farndale RW, Young RD, Barnes MJ. Integrin alpha 2 beta 1 -independent activation of platelets by simple collagen-like peptides: collagen tertiary (triple-helical) and quaternary (polymeric) structures are sufficient alone for alpha 2 beta 1 -independent platelet reactivity. Biochem J 1995; 306(Pt 2):337-344

78 Poulter NS, Pollitt AY, Owen DM, et al. Clustering of glycoprotein VI (GPVI) dimers upon adhesion to collagen as a mechanism to regulate GPVI signaling in platelets. J Thromb Haemost 2017;15 (03):549-564

79 Slater A, Perrella G, Onselaer MB, et al. Does fibrin(ogen) bind to monomeric or dimeric GPVI, or not at all? Platelets 2019;30(03): 281-289

80 Alshehri OM, Hughes CE, Montague S, et al. Fibrin activates GPVI in human and mouse platelets. Blood 2015;126(13):1601-1608

81 Mangin $\mathrm{PH}$, Onselaer M-B, Receveur N, et al. Immobilized fibrinogen activates human platelets through glycoprotein VI. Haematologica 2018;103(05):898-907

82 Riba R, Hughes CE, Graham A, Watson SP, Naseem KM. Globular adiponectin induces platelet activation through the collagen receptor GPVI-Fc receptor $\gamma$ chain complex. JThromb Haemost 2008;6(06):1012-1020

83 Inoue O, Suzuki-Inoue K, McCarty OJT, et al. Laminin stimulates spreading of platelets through integrin alpha6beta1-dependent activation of GPVI. Blood 2006;107(04):1405-1412

84 Inoue O, Suzuki-Inoue K, Ozaki Y. Redundant mechanism of platelet adhesion to laminin and collagen under flow: involvement of von Willebrand factor and glycoprotein Ib-IX-V. J Biol Chem 2008;283(24):16279-16282

85 Schaff M, Tang C, Maurer E, et al. Integrin $\alpha 6 \beta 1$ is the main receptor for vascular laminins and plays a role in platelet adhesion, activation, and arterial thrombosis. Circulation 2013;128(05):541-552

86 Reth M. Antigen receptor tail clue. Nature 1989;338(6214): 383-384

87 Boulaftali Y, Hess PR, Kahn ML, Bergmeier W. Platelet ITAM signaling and vascular integrity. Circ Res 2014;114(07):1174-1184

88 Rayes J, Watson SP, Nieswandt B. Functional significance of the platelet immune receptors GPVI and CLEC-2. J Clin Invest 2019; 129(01):12-23

89 Boylan B, Gao C, Rathore V, Gill JC, Newman DK, Newman PJ. Identification of FcgammaRIIa as the ITAM-bearing receptor mediating alphallbbeta3 outside-in integrin signaling in human platelets. Blood 2008;112(07):2780-2786

90 Watson SP, Auger JM, McCarty OJT, Pearce AC. GPVI and integrin $\alpha$ IIb $\beta 3$ signaling in platelets. JThromb Haemost 2005;3(08): 1752-1762 
91 Locke D, Chen H, Liu Y, Liu C, Kahn ML. Lipid rafts orchestrate signaling by the platelet receptor glycoprotein VI. JBiol Chem 2002;277(21):18801-18809

92 Zhang W, Trible RP, Samelson LE. LAT palmitoylation: its essential role in membrane microdomain targeting and tyrosine phosphorylation during T cell activation. Immunity 1998;9(02):239-246

93 Quinter PG, Dangelmaier CA, Quinton TM, Kunapuli SP, Daniel JL. Glycoprotein VI agonists have distinct dependences on the lipid raft environment. J Thromb Haemost 2007;5(02):362-368

94 Schmaier AA, Zou Z, Kazlauskas A, et al. Molecular priming of Lyn by GPVI enables an immune receptor to adopt a hemostatic role. Proc Natl Acad Sci U S A 2009;106(50):21167-21172

95 Quek LS, Pasquet JM, Hers I, et al. Fyn and Lyn phosphorylate the Fc receptor gamma chain downstream of glycoprotein VI in murine platelets, and Lyn regulates a novel feedback pathway. Blood 2000;96(13):4246-4253

96 Asazuma N, Wilde JI, Berlanga O, et al. Interaction of linker for activation of $\mathrm{T}$ cells with multiple adapter proteins in platelets activated by the glycoprotein VI-selective ligand, convulxin. J Biol Chem 2000;275(43):33427-33434

97 Gross BS, Lee JR, Clements JL, et al. Tyrosine phosphorylation of SLP-76 is downstream of Syk following stimulation of the collagen receptor in platelets. JBiol Chem 1999;274(09): 5963-5971

98 Hughes CE, Auger JM, McGlade J, Eble JA, Pearce AC, Watson SP. Differential roles for the adapters Gads and LAT in platelet activation by GPVI and CLEC-2. JThromb Haemost 2008;6(12): 2152-2159

99 Zhou Z, Gushiken FC, Bolgiano D, et al. Signal transducer and activator of transcription 3 (STAT3) regulates collagen-induced platelet aggregation independently of its transcription factor activity. Circulation 2013;127(04):476-485

100 Oda A, Ikeda Y, Ochs HD, et al. Rapid tyrosine phosphorylation and activation of Bruton's tyrosine/Tec kinases in platelets induced by collagen binding or CD32 cross-linking. Blood 2000;95(05):1663-1670

101 Atkinson BT, Ellmeier W, Watson SP. Tec regulates platelet activation by GPVI in the absence of Btk. Blood 2003;102(10): 3592-3599

102 Pleines I, Elvers M, Strehl A, et al. Rac1 is essential for phospholipase C-gamma2 activation in platelets. Pflugers Arch 2009;457 (05):1173-1185

103 Pearce AC, Senis YA, Billadeau DD, Turner M, Watson SP, Vigorito E. Vav1 and vav3 have critical but redundant roles in mediating platelet activation by collagen. JBiol Chem 2004;279(52): 53955-53962

104 Manganaro D, Consonni A, Guidetti GF, et al. Activation of phosphatidylinositol 3-kinase $\beta$ by the platelet collagen receptors integrin $\alpha 2 \beta 1$ and GPVI: The role of Pyk2 and c-Cbl. Biochim Biophys Acta 2015;1853(08):1879-1888

105 Gilio K, Munnix ICA, Mangin P, et al. Non-redundant roles of phosphoinositide 3-kinase isoforms $\alpha$ and $\beta$ in glycoprotein VIinduced platelet signaling and thrombus formation. J Biol Chem 2009;284(49):33750-33762

106 Auger JM, Best D, Snell DC, Wilde JI, Watson SP. c-Cbl negatively regulates platelet activation by glycoprotein VI. JThromb Haemost 2003;1(11):2419-2426

107 Yacoub D, Théorêt J-F, Villeneuve L, et al. Essential role of protein kinase $C$ delta in platelet signaling, alpha IIb beta 3 activation, and thromboxane A2 release. J Biol Chem 2006;281(40):30024-30035

108 Senis YA, Tomlinson MG, Ellison S, et al. The tyrosine phosphatase CD148 is an essential positive regulator of platelet activation and thrombosis. Blood 2009;113(20):4942-4954

109 Ming Z, Hu Y, Xiang J, Polewski P, Newman PJ, Newman DK. Lyn and PECAM-1 function as interdependent inhibitors of platelet aggregation. Blood 2011;117(14):3903-3906

110 Colonna M, Samaridis J, Angman L. Molecular characterization of two novel C-type lectin-like receptors, one of which is selectively expressed in human dendritic cells. Eur J Immunol 2000;30(02): 697-704

111 Suzuki-Inoue K, Fuller GLJ, García A, et al. A novel Syk-dependent mechanism of platelet activation by the C-type lectin receptor CLEC-2. Blood 2006;107(02):542-549

112 Suzuki-Inoue K, Kato Y, Inoue O, et al. Involvement of the snake toxin receptor CLEC-2, in podoplanin-mediated platelet activation, by cancer cells. J Biol Chem 2007;282(36):25993-26001

113 Christou CM, Pearce AC, Watson AA, et al. Renal cells activate the platelet receptor CLEC-2 through podoplanin. Biochem J 2008; 411(01):133-140

114 Astarita JL, Acton SE, Turley SJ. Podoplanin: emerging functions in development, the immune system, and cancer. Front Immunol 2012;3:283

115 May F, Hagedorn I, Pleines I, et al. CLEC-2 is an essential plateletactivating receptor in hemostasis and thrombosis. Blood 2009; 114(16):3464-3472

116 Hughes CE, Navarro-Núñez L, Finney BA, Mourão-Sá D, Pollitt AY, Watson SP. CLEC-2 is not required for platelet aggregation at arteriolar shear. J Thromb Haemost 2010;8(10):2328-2332

117 Suzuki-Inoue K, Inoue O, Ding G, et al. Essential in vivo roles of the C-type lectin receptor CLEC-2: embryonic/neonatal lethality of CLEC-2-deficient mice by blood/lymphatic misconnections and impaired thrombus formation of CLEC-2-deficient platelets. J Biol Chem 2010;285(32):24494-24507

118 Hatakeyama K, Kaneko MK, Kato Y, et al. Podoplanin expression in advanced atherosclerotic lesions of human aortas. Thromb Res 2012;129(04):e70-e76

119 Hughes CE, Pollitt AY, Mori J, et al. CLEC-2 activates Syk through dimerization. Blood 2010;115(14):2947-2955

120 Fuller GLJ, Williams JAE, Tomlinson MG, et al. The C-type lectin receptors CLEC-2 and Dectin-1, but not DC-SIGN, signal via a novel YXXL-dependent signaling cascade. J Biol Chem 2007;282 (17):12397-12409

121 Judd BA, Myung PS, Obergfell A, et al. Differential requirement for LAT and SLP-76 in GPVI versus T cell receptor signaling. J Exp Med 2002;195(06):705-717

122 Pollitt AY, Grygielska B, Leblond B, Désiré L, Eble JA, Watson SP. Phosphorylation of CLEC-2 is dependent on lipid rafts, actin polymerization, secondary mediators, and Rac. Blood 2010;115 (14):2938-2946

123 Bender M, May F, Lorenz V, et al. Combined in vivo depletion of glycoprotein VI and C-type lectin-like receptor 2 severely compromises hemostasis and abrogates arterial thrombosis in mice. Arterioscler Thromb Vasc Biol 2013;33(05):926-934

124 Chang $\mathrm{CH}$, Chung $\mathrm{CH}$, Tu YS, et al. Trowaglerix venom polypeptides as a novel antithrombotic agent by targeting immunoglobulin-like domains of glycoprotein VI in platelet. Arterioscler Thromb Vasc Biol 2017;37(07):1307-1314

125 Hsu CC, Wu WB, Huang TF. A snake venom metalloproteinase, kistomin, cleaves platelet glycoprotein VI and impairs platelet functions. JThromb Haemost 2008;6(09):1578-1585

126 Fuentes M, Sepúlveda C, Alarcón M, Palomo I, Fuentes E. Buddleja globosa (matico) prevents collagen-induced platelet activation by decreasing phospholipase C-gamma 2 and protein kinase C phosphorylation signaling.J Tradit Complement Med 2017;8(01):66-71

127 Li W, Tang X, Yi W, et al. Glaucocalyxin A inhibits platelet activation and thrombus formation preferentially via GPVI signaling pathway. PLoS One 2013;8(12):e85120

128 Fuentes EJ, Astudillo LA, Gutiérrez MI, et al. Fractions of aqueous and methanolic extracts from tomato (Solanum lycopersicum L.) present platelet antiaggregant activity. Blood Coagul Fibrinolysis 2012;23(02):109-117

129 Hsiao G, Wang Y, Tzu N-H, et al. Inhibitory effects of lycopene on in vitro platelet activation and in vivo prevention of thrombus formation. J Lab Clin Med 2005;146(04):216-226

130 Yoshida S, Sudo T, Niimi M, et al. Inhibition of collagen-induced platelet aggregation by anopheline antiplatelet protein, a saliva 
protein from a malaria vector mosquito. Blood 2008;111(04): 2007-2014

131 Yao Y, Chen Y, Adili R, et al. Plant-based food cyanidin-3-glucoside modulates human platelet glycoprotein VI signaling and inhibits platelet activation and thrombus formation. J Nutr 2017; 147(10):1917-1925

132 Massberg S, Konrad I, Bültmann A, et al. Soluble glycoprotein VI dimer inhibits platelet adhesion and aggregation to the injured vessel wall in vivo. FASEB J 2004;18(02):397-399

133 Bültmann A, Herdeg C, Li Z, et al. Local delivery of soluble platelet collagen receptor glycoprotein VI inhibits thrombus formation in vivo. Thromb Haemost 2006;95(05):763-766

134 Ungerer M, Rosport K, Bültmann A, et al. Novel antiplatelet drug revacept (Dimeric Glycoprotein VI-Fc) specifically and efficiently inhibited collagen-induced platelet aggregation without affecting general hemostasis in humans. Circulation 2011;123(17): 1891-1899

135 Takayama H, Hosaka Y, Nakayama K, et al. A novel antiplatelet antibody therapy that induces cAMP-dependent endocytosis of the GPVI/Fc receptor gamma-chain complex. JClin Invest 2008; 118(05):1785-1795

136 Muzard J, Bouabdelli M, Zahid M, et al. Design and humanization of a murine scFv that blocks human platelet glycoprotein VI in vitro. FEBS J 2009;276(15):4207-4222

137 Matsumoto Y, Takizawa H, Nakama K, et al. Ex vivo evaluation of anti-GPVI antibody in cynomolgus monkeys: dissociation between anti-platelet aggregatory effect and bleeding time. Thromb Haemost 2006;96(02):167-175

138 Li H, Lockyer S, Concepcion A, et al. The Fab fragment of a novel anti-GPVI monoclonal antibody, OM4, reduces in vivo thrombosis without bleeding risk in rats. Arterioscler Thromb Vasc Biol 2007;27(05):1199-1205

139 Florian P, Wonerow P, Harder S, Kuczka K, Dubar M, Graff J. AntiGPVI Fab SAR264565 effectively blocks GPVI function in ex vivo human platelets under arterial shear in a perfusion chamber. Eur J Clin Pharmacol 2017;73(08):949-956

140 Jung SM, Tsuji K, Moroi M. Glycoprotein (GP) VI dimer as a major collagen-binding site of native platelets: direct evidence obtained with dimeric GPVI-specific Fabs. JThromb Haemost 2009;7(08):1347-1355

141 Lebozec K, Jandrot-Perrus M, Avenard G, Favre-Bulle O, Billiald P. Design, development and characterization of ACT017, a humanized Fab that blocks platelet's glycoprotein VI function without causing bleeding risks. MAbs 2017;9(06):945-958

142 O'Connor MN, Smethurst PA, Davies LW, et al. Selective blockade of glycoprotein VI clustering on collagen helices. JBiol Chem 2006;281(44):33505-33510

143 Uckun FM, Vassilev A, Bartell S, Zheng Y, Mahajan S, Tibbles HE. The anti-leukemic Bruton's tyrosine kinase inhibitor alpha-cyano-beta-hydroxy-beta-methyl-N-(2,5-dibromophenyl) propenamide (LFM-A13) prevents fatal thromboembolism. Leuk Lymphoma 2003;44(09):1569-1577

144 Spalton JC, Mori J, Pollitt AY, Hughes CE, Eble JA, Watson SP. The novel Syk inhibitor R406 reveals mechanistic differences in the initiation of GPVI and CLEC-2 signaling in platelets. JThromb Haemost 2009;7(07):1192-1199

145 Reilly MP, Sinha U, André P, et al. PRT-060318, a novel Syk inhibitor, prevents heparin-induced thrombocytopenia and thrombosis in a transgenic mouse model. Blood 2011;117(07): 2241-2246

146 Spyridon M, Moraes LA, Jones CI, et al. LXR as a novel antithrombotic target. Blood 2011;117(21):5751-5761

147 Bishton MJ, Gardiner EE, Harrison SJ, Prince HM, Johnstone RW. Histone deacetylase inhibitors reduce glycoprotein VI expression and platelet responses to collagen related peptide. Thromb Res 2013;131(06):514-520

148 Vara D, Campanella M, Pula G. The novel NOX inhibitor 2acetylphenothiazine impairs collagen-dependent thrombus formation in a GPVI-dependent manner. Br J Pharmacol 2013;168 (01):212-224

149 Grothusen C, Umbreen S, Konrad I, et al. EXP3179 inhibits collagen-dependent platelet activation via glycoprotein receptor-VI independent of AT1-receptor antagonism: potential impact on atherothrombosis. Arterioscler Thromb Vasc Biol 2007;27(05):1184-1190

150 Lin K-H, Lu W-J, Wang S-H, et al. Characteristics of endogenous $\gamma-$ aminobutyric acid (GABA) in human platelets: functional studies of a novel collagen glycoprotein VI inhibitor. J Mol Med (Berl) 2014;92(06):603-614

151 Kamiguti AS. Platelets as targets of snake venom metalloproteinases. Toxicon 2005;45(08):1041-1049

152 Fuentes E, Fuentes F, Palomo I. Mechanism of the anti-platelet effect of natural bioactive compounds: role of peroxisome proliferator-activated receptors activation. Platelets 2014;25(07): 471-479

153 Akbiyik F, Ray DM, Gettings KF, Blumberg N, Francis CW, Phipps RP. Human bone marrow megakaryocytes and platelets express PPARgamma, and PPARgamma agonists blunt platelet release of CD40 ligand and thromboxanes. Blood 2004;104(05): 1361-1368

154 Moraes LA, Spyridon M, Kaiser WJ, et al. Non-genomic effects of PPARgamma ligands: inhibition of GPVI-stimulated platelet activation. J Thromb Haemost 2010;8(03):577-587

155 Reimann A, Li Z, Goebel S, et al. Combined administration of the GPVI-Fc fusion protein Revacept with low-dose thrombolysis in the treatment of stroke. Heart Int 2016;11(01):e10-e16

156 Nieswandt B, Bergmeier W, Schulte V, Rackebrandt K, Gessner JE, Zirngibl $\mathrm{H}$. Expression and function of the mouse collagen receptor glycoprotein VI is strictly dependent on its association with the FcRgamma chain. JBiol Chem 2000;275(31): 23998-24002

157 Stegner D, Haining EJ, Nieswandt B. Targeting glycoprotein VI and the immunoreceptor tyrosine-based activation motif signaling pathway. Arterioscler Thromb Vasc Biol 2014;34(08): $1615-1620$

158 Martins Lima A, Bragina ME, Burri O, et al. An optimized and validated 384-well plate assay to test platelet function in a highthroughput screening format. Platelets 2019;30(05):563-571 